\title{
Event-triggered neuroadaptive output-feedback control for nonstrict-feedback nonlinear systems with given performance specifications
}

\author{
Di Yang · Weijun Liu · Chen Guo
}

Received: date / Accepted: date

\begin{abstract}
This paper focuses on the event-triggered neuroadaptive output-feedback tracking control issue for nonstrictfeedback nonlinear systems with given performance specifications. By constructing a neural observer to estimate unmeasurable states, a novel event-triggered controller is presented together with a piecewise threshold rule. The presented event-triggered mechanism has two thresholds to reduce communication resources between the controller and actuator. An improved speed transformation function is introduced to make the output tracking error converge to a preassigned small region at predesigned converging mode within preset finite time. Combining the variable separation method based on the structural property of radial basis function (RBF) and backstepping technology, the algebraic loop problem caused by the nonstrict-feedback structure is overcome. The command filtered technology with filtering error compensating signal is applied to address the "explosion of complexity" problem. Furthermore, Lyapunov stability analysis demonstrates that under the presented eventtriggered controller, all signals in the closed-loop system are semiglobally bounded, and the Zeno-behaviour is ruled out
\end{abstract}

D. Yang $\cdot$ W. Liu

School of Mechanical Engineering, Shenyang University of Technology, Shenyang 110870, Liaoning, People's Republic of China e-mail: yyaaid@163.com

W. Liu (四)

e-mail: wjliu@sut.edu.cn

\section{Yang}

School of Chemical Process Automation, Shenyang University of Technology, Liaoyang 111003, Liaoning, People's Republic of China

C. Guo

School of Marine Electrical Engineering, Dalian Maritime University, Dalian 116026, Liaoning, People's Republic of China

e-mail: guocarticle@163.com strictly. Numerical simulations are finally provided to illustrate the presented control scheme.

Keywords Nonstrict-feedback nonlinear systems · Adaptive neural control - Given performance specifications · Speed transformation function - Event-triggered control · Command filtered backstepping

\section{Introduction}

Adaptive backstepping technology has become a resultful instrument to design the control scheme for uncertain strictfeedback systems, and a lot of significant research results have been achieved (see [1]-[7]). Whereas many practical engineering systems, such as the helicopter model [8], the ball and beam system [9], and uncertain robot systems [10], are in nonstrict-feedback form. The algebraic loop problem, caused by the nonstrict-feedback structure, will render the traditional adaptive backstepping control method discussed in strict-feedback systems invalid. Thus, to solve this problem, the Butterworth low-pass filter was adopted in [11]-[12] for pure-feedback nonlinear systems. During the control law design process, it is known that the introduction of additional low-pass filter will inevitably lead to the increase in algorithm complexity. Without introducing the additional filter, some adaptive neural networks/fuzzy control strategies were developed in [13]-[16] for nonstrict-feedback nonlinear systems via utilizing the variable separation technology. The restrictive condition, requiring the unknown functions to conform to the monotonically increasing property [13]-[16], was overcome in [17]-[18] according to the structual property of fuzzy-logic systems or neural networks. Such results were further generalized to the cases of prescribed performance control and finite-time control. To ensure that the tracking error enters into a prescribed range, an adaptive fuzzy controller was constructed in [19] for nontriangular 
nonlinear systems, in which the tracking error with constraint was converted into a new variable. Via applying a fuzzy observer to estimate unmeasurable states, authors in [20] investigated the finite-time adaptive control issue for nonlinear systems in nontriangular form, where the dynamic surface control scheme was adopted to overcome the "explosion of complexity" problem resulted from the iterative differentiations for virtual control functions.

Although the prescribed performance control can achieve that the output error enters into a predefined compact set at the prescribed converging mode [21]-[23], it cannot guarantee that the convergence of output error is realized in a finite time. In order for the tracking error to converge within a finite time, the finite-time controller in [24]-[26] attempts to give a new idea, but the settling time, depending on the initial condition of system states, cannot be given arbitrarily and chosen in advance. Fortunately, the work [27] applied a speed function to transform the original output error into an accelerated one, and then the convergence performance of original output error can be adjusted via stabilizing the accelerated dynamic system. This idea was further extended in [28] to design prescribed performance controller for MIMO strict-feedback systems, where the tracking error can converge to a preassigned compact set in a pre-given time. More recently, via blending finite-time performance function and intermediate transformation, the finite-time controller with prescribed performance was presented for nonlinear strictfeedback systems in [29]-[31] and for nonlinear nonstrictfeedback systems in [32], respectively. However, the results presented in [27]-[32] are dependent on the full-state information, and do not take into account the event-triggered control issue.

Due to the increasing popularity of the network control, event-triggered scheme has been widely acknowledged as an effective alternative to traditional periodic sampling scheme, which can greatly reduce transmission burden in the communication network. Thus, some significant results about event-triggered control scheme can be found in [33]-[35]. Among them, the authors in [33] developed an output-feedback controller with prescribed performance for pure-feedback systems using the fixed threshold event-triggered mechanism. It should be noted that the threshold value in [33] does not change during the control process. For further decreasing the data transmission and increasing the efficiency in resource utilization, an event-triggered adaptive controller was designed in [34] for strict-feedback systems with actuator failures by utilizing the neural state observer, where a varying threshold method, in the light of the value of control signal, was proposed to update the control signal. Based on the linear state observer, an event-triggered adaptive neural network controller was proposed in [35] for nonstrict-feedback systems with unknown control directions via adopting relative threshold strategy. However, the complexity of the event- triggered control algorithm based on relative threshold will increase in order to guarantee the stability of whole system because the triggering condition contains the control signal.

According to the discussions above, it is very necessary to develop a new event-triggered neuroadaptive outputfeedback controller with given performance specifications for nonstrict-feedback uncertain nonlinear systems, which drives the current work. Specifically, there are three complicated problems to be solved in the article:

1) How to use a speed transformation function to design an event-triggered output-feedback controller with given performance specifications for nonstrict-feedback uncertain nonlinear systems?

2) How to effectively reduce data transmission and improve resource utilization while avoiding the inclusion of control signal in the triggering condition?

3) How to introduce command filtering technology with filtering error compensating signal to deal with the "explosion of complexity" problem during the control strategy design process?

In this work, a new event-triggered adaptive control scheme combined with neural state observer and command filtering technology will be investigated for nonstrict-feedback uncertain nonlinear systems with given performance specifications. Furthermore, the variable separation method and backstepping technology will be adopted to deal with the algebraic loop problem arising from nonstrict-feedback structure. The main features and contributions of the proposed controller lie in the following aspects:

1) In comparison to the observer-based finite-time adaptive tracking control in [4] and [20], observer-based prescribed performance tracking control in [36], the proposed output-feedback control scheme can ensure that not only the tracking error enters into the prescribed bounded set within a preset time, but also decay rate during the preset time interval is predesigned and controllable explicitly. Further, the preset time is independent of the initial condition of system states.

2) In comparison to the speed transformation functionbased tracking control strategy with given performance specifications in [27] and [28], finite-time tracking control strategy with prescribed performance in [31] for strict-feedback nonlinear systems, this paper applies the output information and universal approximation ability of neural networks to construct state observer, and a novel event-triggered tracking controller is presented for more general nonstrict-feedback nonlinear systems.

3) Compared with the event-triggered prescribed performance controller using the fixed threshold strategy in [33] for pure-feedback nonlinear systems, the designed eventtriggered mechanism has two thresholds, which can effectively reduce the data transmission and avoid including the control signal in the trigger condition. Furthermore, the com- 
mand filtering technology is employed to deal with the "explosion of complexity" and "filtering error compensation" problem by utilizing filtering error compensating signal.

\section{Problem formulation}

\subsection{System description}

Consider the following uncertain nonstrict-feedback system:

$$
\left\{\begin{array}{l}
\dot{x}_{k}=x_{k+1}+h_{k}(X), k=1,2, \ldots, n-1 \\
\dot{x}_{n}=u+h_{n}(X) \\
y=x_{1}
\end{array}\right.
$$

where $X=\left[x_{1}, \ldots, x_{n}\right]^{\mathrm{T}} \in R^{n}$ and $u \in R$ represent plant state vector and control input, respectively. $y \in R$ is the output of plant. $h_{k}(X), k=1,2, \ldots, n$ represent unknown smooth nonlinear functions. It is assumed that nonstrict-feedback system (1) is completely observable and controllable and only system output $y$ can be directly measured.

Remark 1 Because the nonlinear function $h_{k}(X)$ in each subsystem contains the whole plant state vector $X=\left[x_{1}, \ldots, x_{n}\right]^{\mathrm{T}}$, the plant (1) is a nonstrict-feedback system. It is worth noting that the control methods developed in [5] and [31] are effective for strict-feedback systems with full-state information. If the backstepping technology in [5] and [31] is adopted to develop control law for nonstrict-feedback system (1), the algebraic loop problem will arise, making it very difficult to design virtual controller. In practice, many practical plants are with nonstrict-feedback structure and unmeasured states. Therefore, the system (1) considered in the article is very necessary.

The purpose of this paper is to present an event-triggered neuroadaptive output-feedback control scheme with given performance specifications for (1), such that:

1) All signals in the closed-loop system are semiglobally bounded.

2) The tracking error $\chi_{1}=y-y_{d}$, where $y_{d}$ is the desired reference signal, converges to a prescribed compact set at predesigned decay rate within a pre-given finite time.

3) A novel event-triggered mechanism is designed to effectively reduce data transmission between the controller and actuator and the Zeno behaviour is strictly ruled out.

To achieve the control objective, we require the following assumption and lemmas.

Assumption 1 The desired reference signal $y_{d}$ and its time derivative $\dot{y}_{d}$ are smooth, bounded, and available.

Remark 2 Compared with traditional backstepping method, the control scheme in this article does not have strict requirements on the desired reference signal, because only the information of $y_{d}$ and $\dot{y}_{d}$ is used in the design process.
Lemma 1 [37] For hyperbolic tangent function $\tanh (\cdot)$, the following property holds

$0 \leq-\hbar \tanh \left(\frac{\hbar}{\bar{\varpi}}\right)+|\hbar| \leq 0.2785 \varpi$

where $\varpi>0, \hbar \in R,-\hbar \tanh (\hbar / \varpi) \leq 0$.

Lemma 2 [38] Consider a continuous nonlinear function $h(X)$ defined on a compact set $\Omega$. Then for $\forall \varepsilon>0$, there exists a RBF neural network satisfying

$\sup _{x \in \Omega}\left|h(X)-\theta^{T} \phi(X)\right| \leq \varepsilon$

where $\theta=\left[\theta_{1}, \ldots, \theta_{M}\right]^{T}$ represents the ideal weight vector, $\phi(X)=\left[p_{1}(X), \ldots, p_{M}(X)\right]^{T}$ denotes the basis function vector, $M>1$ is node number of neural network, and $p_{k}(X)$ is constructed as $p_{k}(X)=\exp \left[-\left(X-v_{k}\right)^{T}\left(X-v_{k}\right) / \gamma_{k}^{2}\right], k=$ $1,2, \ldots, M, v_{k}=\left[v_{k 1}, \ldots, v_{k n}\right]^{T}$ is the center vector and $\gamma_{k}$ is the width of Gaussian function.

Remark 3 According to the definition of $p_{k}(X)$ and $\phi(X)$, it can be demonstrated that $0<p_{k}(X) \leq 1$ and $0<\phi(X)^{\mathrm{T}} \phi(X)$ $\leq M$, the characteristic of which will be utilized to separate the whole state variable in the system function $h_{k}(X)$.

\subsection{Speed function}

Inspired by [27] and [28], an improved speed function is introduced as follows:

$\mu(t)=\left\{\begin{array}{l}\frac{t_{r}^{3}}{\left(1-\mu_{t r}\right)\left(t_{r}-t\right)^{3} \mu_{0}+\mu_{0} \mu_{t r} t_{r}^{3}}, t \in\left[0, t_{r}\right) \\ \frac{1}{\mu_{0} \mu_{t r}}, \quad t \in\left[t_{r},+\infty\right)\end{array}\right.$

where $\mu_{0}$ and $\mu_{t r}<1$ are positive parameters, $t_{r}>0$ and $\mu_{0} \mu_{t r}$ denote finite settling time and ultimate bound of tracking error, respectively.

Lemma 3 [28] The unique properties of speed function in (4) can be described as follows.

1) For $t \in\left[0, t_{r}\right), \mu(t)$ is strictly increasing with $\mu(0)=$ $1 / \mu_{0}$ and $\mu \in\left[1 / \mu_{0}, 1 / \mu_{0} \mu_{t r}\right]$ for $t \in[0,+\infty)$.

2) At $t=t_{r}, \mu(t)$ reaches its maximum value $1 / \mu_{0} \mu_{t r}$ and remains to be $1 / \mu_{0} \mu_{\text {tr }}$ for $t \in\left[t_{r},+\infty\right)$.

3) For $t \in[0,+\infty), \mu^{(i)}(t)(i=0,1,2)$ are $C^{2-i}$ and bounded, and $\mu^{-1}(t) \dot{\mu}(t)$ is bounded.

Remark 4 Although some prescribed performance controllers, using exponential performance function $\rho(t)=\left(\rho_{0}-\rho_{\infty}\right) e^{-\omega t}$ $+\rho_{\infty}($ see $[21]-[23])$, have been developed to deal with the tracking error, they cannot guarantee that the tracking error enters into a preassigned compact set within a known time. However, the speed function considered in this article can make the convergence of tracking error have the given performance specifications, which is more challenging and difficult. 


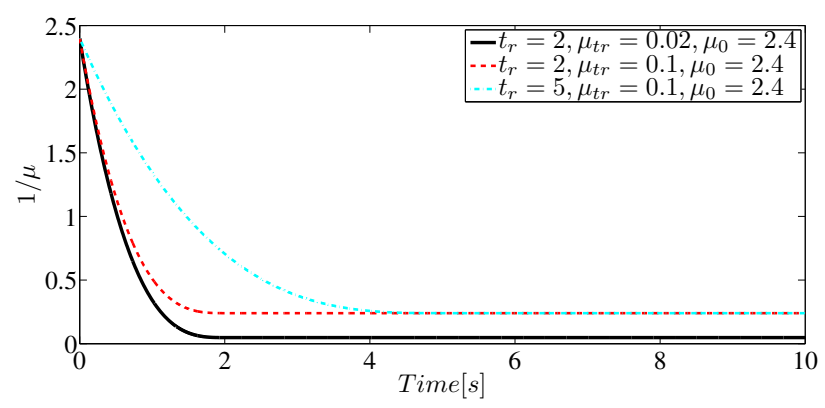

Fig. 1 Trajectories of $1 / \mu$ in different cases.

To achieve the pregiven performance specifications, the following state transformation is defined:

$s_{1}=\tan \left(\frac{\pi}{2} \mu \chi_{1}\right), \chi_{1}(0)<\mu_{0}$

From the definition of (5), we have

$\chi_{1}=\frac{2}{\pi \mu} \operatorname{actan} s_{1}$

and

$\dot{\chi}_{1}=-\frac{2 \dot{\mu}}{\pi \mu^{2}} \arctan s_{1}+\frac{2}{\pi \mu} \frac{\dot{s}_{1}}{1+s_{1}^{2}}$

$=x_{2}+h_{1}(X)-\dot{y}_{d}$

which yields

$\dot{s}_{1}=\Xi x_{2}+\Xi h_{1}(X)-\Xi \dot{y}_{d}+\Xi \frac{2 \dot{\mu}}{\pi \mu^{2}} \arctan s_{1}$

where $\Xi=\pi \mu\left(1+s_{1}^{2}\right) / 2>0$.

Remark 5 In contrast to the speed function in [27] and [28], a new parameter $\mu_{0}$ is introduced to obtain $\mu(0)=\mu_{0}$ rather than $\mu(0)=1$, in which the restrictive condition of the initial tracking error $\chi_{1}(0)$ is relaxed largely. In addition, different from the existing state transformation in [39] and [12], a distinguishing feature, via using (5), is to ensure that the value of function $\Xi$ is always positive, which is very necessary to design a stable filtering error compensation system.

Remark 6 The advantage of using state transformation is to convert the constrained tracking error $\chi_{1}$ into an equivalent unconstrained signal. It should be noted from (5) that if $s_{1}$ is bounded, then $-1 / \mu<\chi_{1}<1 / \mu$ where the curve of $1 / \mu$ is shown in Fig 1 . Therefore, by designing the control method to ensure the boundedness of $s_{1}$, the pregiven performance specifications of $\chi_{1}$ can be realized indirectly.

\subsection{Event-triggered mechanism}

Inspired by [33], a novel event-triggered mechanism with two thresholds is designed as follows.

$u(t)=\tau\left(t_{q}\right), t_{q} \leq t<t_{q+1}$

$t_{q+1}=\left\{\begin{array}{l}\inf \left\{t>t_{q}|| \varsigma(t) \mid \geq \rho_{a x}\right\}, \text { if } t<T^{\dagger} \\ \inf \left\{t>t_{q}|| \varsigma(t) \mid \geq \rho_{\text {in }}\right\}, \text { if } t \geq T^{\dagger}\end{array}\right.$

where $\varsigma(t)=\tau(t)-u(t)$ denotes the measurement error between the intermediate control $\tau(t)$ and control input $u(t)$, $\rho_{a x}, \rho_{i n}, T^{\dagger}$ are positive design parameters and $\rho_{a x}>\rho_{i n}, t_{r}>$ $T^{\dagger}, t_{q}, q \in z^{+}$, denotes controller updating moment, i.e., once (10) is triggered, the actuator will be updated by $\tau\left(t_{q+1}\right)$. In the period $t \in\left[t_{q}, t_{q+1}\right)$, the actuator holds as a constant $\tau\left(t_{q}\right)$. During the initial stage $t \in\left[0, T^{\dagger}\right)$, a relatively large threshold is chosen to deal with large control signal caused by initial tracking error to obtain longer event-triggered intervals. In another condition when tracking error gradually comes to stability, the control signal should be close to zero. Thus, to achieve a better system stabilization performance, a smaller measurement error is designed to obtain a more precise control signal.

Remark 7 Compare with fixed threshold event-triggered strategy in [33], the relative threshold method [35] can effectively reduce the number of triggering events because the output of controller is a time-varying signal during system operation. However, the relative threshold method, in which the control signal is introduced into triggering condition, will complicate the expression of the control algorithm. In order to effectively reduce the number of triggering events and avoid introducing the control signal into triggering condition, a novel event-triggered mechanism with two thresholds is designed in this work, whose superiority will be verified in the following comparative simulations.

\section{Control design and stability analysis}

\subsection{Neural state observer design}

Due to unmeasurable state variables and unknown nonlinear functions, the RBF neural networks are utilized to be uncertainty approximators embedded in the observer, and thereby contributing to a neural state observer. Before constructing the neural state observer, the nonstrict-feedback system (1) can be rewritten as

$\left\{\begin{array}{l}\dot{X}=A X+L y+\sum_{k=1}^{n} B_{k} h_{k}(X)+B u \\ y=C X\end{array}\right.$

where 


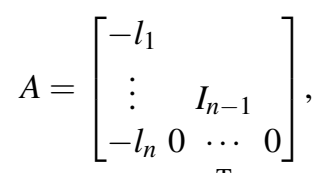

$B_{k}=[\underbrace{0,0, \ldots, 0,1}_{k}, 0, \ldots, 0]^{\mathrm{T}}, B=[0, \ldots, 0,1]^{\mathrm{T}}, L=\left[l_{1}, l_{2}\right.$

$\left., \ldots, l_{n}\right]^{\mathrm{T}}$, and $C=[1,0, \ldots, 0]$. Via selecting proper $L$, a strict

Hurwitz matrix can be obtained. Therefore, given $Q^{\mathrm{T}}=Q>$ 0 , there is a positive definite matrix $P^{\mathrm{T}}=P>0$ satisfying

$$
A^{\mathrm{T}} P+P A=-2 Q \text {. }
$$

Construct a neural observer as follows:

$$
\dot{\hat{X}}=A \hat{X}+L y+\sum_{k=1}^{n} B_{k} \hat{h}_{k}\left(\hat{X} \mid \theta_{k}\right)+B u
$$

where $\hat{X}=\left[\hat{x}_{1}, \ldots, \hat{x}_{n}\right]^{\mathrm{T}}$ are defined to estimate $X=\left[x_{1}, \ldots, x_{n}\right]^{\mathrm{T}}$, and under Lemma 2 , the nonlinear functions in (1) can be estimated as $h_{k}\left(X \mid \theta_{k}\right)=\theta_{k}^{\mathrm{T}} \phi_{k}(X)$ and $\hat{h}_{k}\left(\hat{X} \mid \theta_{k}\right)=\theta_{k}^{\mathrm{T}} \phi_{k}(\hat{X})$.

Similar to [40]-[42], the variable errors $\varepsilon_{k}$ and $\delta_{k}$ are defined as

$$
\left\{\begin{array}{c}
\varepsilon_{k}=h_{k}(X)-\hat{h}_{k}\left(\hat{X} \mid \theta_{k}^{*}\right) \\
\delta_{k}=h_{k}(X)-\hat{h}_{k}\left(\hat{X} \mid \theta_{k}\right)
\end{array}\right.
$$

where $\theta_{k}^{*}$ denotes the optimal parameter vector, and we assume that there exist positive constants $\bar{\varepsilon}_{k}$ and $\bar{\delta}_{k}, k=1,2, \ldots, n$, such that $\left|\varepsilon_{k}\right| \leq \bar{\varepsilon}_{k}$ and $\left|\delta_{k}\right| \leq \bar{\delta}_{k}$.

Denote $\tilde{X}=X-\hat{X}=\left[\tilde{x}_{1}, \ldots, \tilde{x}_{n}\right]^{\mathrm{T}}$ as the observer error. Then from (11) and (13), we can obtain the error dynamics:

$$
\begin{aligned}
\dot{\tilde{X}} & =A \tilde{X}+\sum_{k=1}^{n} B_{k}\left(h_{k}(X)-\hat{h}_{k}\left(\hat{X} \mid \theta_{k}\right)\right) \\
& =A \tilde{X}+\delta
\end{aligned}
$$

where $\delta=\left[\delta_{1}, \ldots, \delta_{n}\right]^{\mathrm{T}}$.

Remark 8 Since only the output $y$ can be used to design the control law, a neural state observer is constructed to estimate unmeasured state vector. Compared with the linear state observer in [43] and [10], the observer (13) uses neural networks to approximate useful information of uncertain function $h_{k}(X)$ for obtaining better estimation performance

\subsection{Event-triggered control design}

To avoid the repeated differentiations virtual controllers in the backstepping design, the following tracking errors and command filtered system are defined:

$z_{1}=s_{1}$

$z_{k}=\hat{x}_{k}-\lambda_{k}, \quad k=2, \ldots, n$

where $\lambda_{k}$ denotes the output of the following command filter with virtual controller $\alpha_{k-1}$

$\omega_{k-1} \dot{\lambda}_{k}+\lambda_{k}=\alpha_{k-1}, \quad \lambda_{k}(0)=\alpha_{k-1}(0), \quad k=2, \ldots, n$ where $\omega_{k-1}$ is a positive design parameter.

The following virtual controllers and actual controller are given for $t \in\left[t_{q}, t_{q+1}\right)$

$$
\begin{aligned}
\alpha_{1}= & -\frac{c_{1} z_{1}}{\Xi}-\frac{a_{13}}{2} \eta_{1} \Xi-a_{11} \eta_{1} \Xi-a_{12} \eta_{1} \Xi \\
& -\theta_{1}^{\mathrm{T}} \phi_{1}\left(\hat{x}_{1}\right)-\frac{2 \dot{\mu}}{\pi \mu^{2}} \arctan s_{1}+\dot{y}_{d} \\
\alpha_{2}= & -c_{2} z_{2}-a_{21} \eta_{2}-\frac{a_{23}}{2} \eta_{2}-\frac{a_{22}}{2} \eta_{2} \\
& -\theta_{2}^{\mathrm{T}} \phi_{2}\left(\hat{X}_{2}\right)-l_{2} \tilde{x}_{1}+\dot{\lambda}_{2}-\Xi z_{1} \\
\alpha_{k}= & -c_{k} z_{k}-a_{k 1} \eta_{k}-\frac{a_{k 3}}{2} \eta_{k}-\frac{a_{k 2}}{2} \eta_{k}-\theta_{k}^{\mathrm{T}} \phi_{k}\left(\hat{X}_{k}\right) \\
& -l_{k} \tilde{x}_{1}+\dot{\lambda}_{k}-z_{k-1}, k=3, \ldots, n-1 \\
\alpha_{n}= & -c_{n} z_{n}-\frac{a_{n 3}}{2} \eta_{n}-\frac{a_{n 2}}{2} \eta_{n}-\theta_{n}^{\mathrm{T}} \phi_{n}(\hat{X})-l_{n} \tilde{x}_{1} \\
& +\dot{\lambda}_{n}-z_{n-1} \\
\tau(t)= & \alpha_{n}-\bar{\rho} \tanh \left(\frac{\eta_{n} \bar{\rho}}{\varpi}\right) \\
u(t)= & \tau\left(t_{q}\right)
\end{aligned}
$$

where $\bar{\rho}>\rho_{a x}>\rho_{i n}, \Phi>0, c_{k}>0, a_{k 2}>0, a_{k 3}>0$ and $a_{j 1}>0, k=1, \ldots, n, j=1, \ldots, n-1$ are design parameters. $\eta_{k}$ denotes the compensated tracking error given as

$\eta_{k}=z_{k}-\xi_{k}, \quad k=1,2, \ldots, n$.

where $\xi_{k}$ is error compensation signal designed in 21). The updating process of parameters can be designed as

$\dot{\theta}_{1}=\pi_{1} \Xi \eta_{1} \phi_{1}\left(\hat{x}_{1}\right)-\sigma_{1} \theta_{1}$

$\dot{\theta}_{k}=\pi_{k} \eta_{k} \phi_{k}\left(\hat{X}_{k}\right)-\sigma_{k} \theta_{k}, k=2, \ldots, n$

where $\hat{X}_{k}=\left[\hat{x}_{1}, \hat{x}_{2}, \ldots, \hat{x}_{k}\right]^{\mathrm{T}}, k=2, \ldots, n, \hat{X}_{n}=\hat{X}, \pi_{k}>0$ and $\sigma_{k}>0$ are design parameters.

To eliminate the adverse effect of filtering errors $\lambda_{k}-$ $\alpha_{k-1}(k=2, \ldots, n)$, we design the error compensation mechanism as

$\dot{\xi}_{1}=-c_{1} \xi_{1}+\Xi \xi_{2}+\Xi\left(\lambda_{2}-\alpha_{1}\right)-\Xi l_{1} \operatorname{sign}\left(\xi_{1}\right)$

$\dot{\xi}_{2}=-c_{2} \xi_{2}-\Xi \xi_{1}+\lambda_{3}-\alpha_{2}+\xi_{3}-\imath_{2} \operatorname{sign}\left(\xi_{2}\right)$

$\dot{\xi}_{k}=-c_{k} \xi_{k}-\xi_{k-1}+\lambda_{k+1}-\alpha_{k}+\xi_{k+1}-\imath_{k} \operatorname{sign}\left(\xi_{k}\right)$

$(k=3, \ldots, n-1)$

$\dot{\xi}_{n}=-c_{n} \xi_{n}-\xi_{n-1}-i_{n} \operatorname{sign}\left(\xi_{n}\right)$

with $\xi_{k}(0)=0(k=1, \ldots, n), \imath_{k}$ is a positive design parameter.

Remark 9 Although the dynamic surface technique was employed in [20] to solve the "explosion of complexity" problem for nonstrict-feedback nonlinear systems, the adverse effect of filtering error $\lambda_{k}-\alpha_{k-1}$ was not addressed, which might degrade control quality. In order to cope with the adverse effect, the error compensation mechanism in 21) is developed at each step of the control strategy. 
3.3 Stability analysis

In this section, the specifics of developed event-triggered control strategy are presented according to the backstepping technique, where the $n$ recursive steps are involved.

Step 1: Based on the compensated tracking errors (19), the derivative of $\eta_{1}$ is given as

$$
\begin{aligned}
\dot{\eta}_{1}= & \dot{z}_{1}-\dot{\xi}_{1} \\
= & \Xi \tilde{x}_{2}+\Xi\left(z_{2}+\lambda_{2}\right)+\Xi\left(\theta_{1}^{*^{\mathrm{T}}} \phi_{1}(\hat{X})+\varepsilon_{1}\right. \\
& \left.-\dot{y}_{d}+\frac{2 \dot{\mu}}{\pi \mu^{2}} \arctan s_{1}\right)-\dot{\xi}_{1} \\
= & \Xi \tilde{x}_{2}+\Xi\left(z_{2}+\lambda_{2}\right)+\Xi \varepsilon_{1}-\dot{\xi}_{1} \\
& +\Xi\left(\theta_{1}^{*^{\mathrm{T}}} \phi_{1}(\hat{X})-\theta_{1}^{*^{\mathrm{T}}} \phi_{1}\left(\hat{x}_{1}\right)\right)+\Xi \theta_{1}^{\mathrm{T}} \phi_{1}\left(\hat{x}_{1}\right) \\
& +\Xi \tilde{\theta}_{1}^{\mathrm{T}} \phi_{1}\left(\hat{x}_{1}\right)+\Xi\left(\frac{2 \dot{\mu}}{\pi \mu^{2}} \arctan s_{1}-\dot{y}_{d}\right)
\end{aligned}
$$

where $\tilde{\theta}_{1}=\theta_{1}^{*}-\theta_{1}$. Constructing the Lyapunov function

$V_{1}=V_{\tilde{x}}+\frac{1}{2} \eta_{1}^{2}+\frac{1}{2 \pi_{1}} \tilde{\theta}_{1}^{2}$

where $V_{\tilde{x}}=\tilde{X}^{\mathrm{T}} P \tilde{X} / 2$. Its time derivative is obtained as

$$
\begin{aligned}
\dot{V}_{1}= & \dot{V}_{\tilde{x}}+\eta_{1} \dot{\eta}_{1}-\frac{1}{\pi_{1}} \tilde{\theta}_{1}^{\mathrm{T}} \dot{\theta}_{1} \\
= & -\tilde{X}^{\mathrm{T}} Q \tilde{X}+\tilde{X}^{\mathrm{T}} P \delta+\eta_{1}\left(\Xi \tilde{x}_{2}+\Xi \eta_{2}+\Xi \alpha_{1}\right. \\
& +\Xi\left(\theta_{1}^{*^{\mathrm{T}}} \phi_{1}(\hat{X})-\theta_{1}^{*^{\mathrm{T}}} \phi_{1}\left(\hat{x}_{1}\right)\right)+\Xi \theta_{1}^{\mathrm{T}} \phi_{1}\left(\hat{x}_{1}\right) \\
& +\Xi \tilde{\theta}_{1}^{\mathrm{T}} \phi_{1}\left(\hat{x}_{1}\right)+\Xi \varepsilon_{1}+\Xi\left(\frac{2 \dot{\mu}}{\pi \mu^{2}} \arctan s_{1}-\dot{y}_{d}\right) \\
& \left.+c_{1} \xi_{1}+\Xi l_{1} \operatorname{sign}\left(\xi_{1}\right)\right)-\frac{1}{\pi_{1}} \tilde{\theta}_{1}^{\mathrm{T}} \dot{\theta}_{1}
\end{aligned}
$$

By Young's inequality, and applying the property of RBF, that is $0<\phi_{1}(\cdot)^{\mathrm{T}} \phi_{1}(\cdot) \leq M_{1}$, one has

$\eta_{1} \Xi\left(\theta_{1}^{*^{\mathrm{T}}} \phi_{1}(\hat{X})-\theta_{1}^{*^{\mathrm{T}}} \phi_{1}\left(\hat{x}_{1}\right)\right) \leq a_{11} \eta_{1}^{2} \Xi^{2}+\frac{\left\|\theta_{1}^{*}\right\|^{2} M_{1}}{a_{11}}$

$\eta_{1} \Xi \varepsilon_{1}+\eta_{1} \Xi \iota_{1} \operatorname{sign}\left(\xi_{1}\right) \leq a_{12} \eta_{1}^{2} \Xi^{2}+\frac{\bar{\varepsilon}_{1}^{2}}{2 a_{12}}+\frac{\imath_{1}^{2}}{2 a_{12}}$

$\eta_{1} \Xi \tilde{x}_{2} \leq \frac{a_{13}}{2} \eta_{1}^{2} \Xi^{2}+\frac{\|\tilde{X}\|^{2}}{2 a_{13}}$

$\tilde{X}^{\mathrm{T}} P \delta \leq \frac{a_{14}}{2}\|P \delta\|^{2}+\frac{\|\tilde{X}\|^{2}}{2 a_{14}}$
Substituting (25)-28) into 24, yields

$$
\begin{aligned}
\dot{V}_{1} \leq & -\tilde{X}^{\mathrm{T}}\left(\lambda_{\min }(Q)-\frac{1}{2 a_{13}}-\frac{1}{2 a_{14}}\right) \tilde{X}+\Xi \eta_{1} \eta_{2} \\
& +\Xi \eta_{1} \tilde{\theta}_{1}^{\mathrm{T}} \phi_{1}\left(\hat{x}_{1}\right)-\frac{1}{\pi_{1}} \tilde{\theta}_{1}^{\mathrm{T}} \dot{\theta}_{1}+\eta_{1} \Xi\left(\alpha_{1}\right. \\
& +\frac{a_{13}}{2} \eta_{1} \Xi+a_{11} \eta_{1} \Xi+a_{12} \eta_{1} \Xi+\theta_{1}^{\mathrm{T}} \phi_{1}\left(\hat{x}_{1}\right) \\
& \left.+\frac{2 \dot{\mu}}{\pi \mu^{2}} \arctan s_{1}-\dot{y}_{d}+\frac{c_{1} \xi_{1}}{\Xi}\right)+\frac{a_{14}}{2}\|P \delta\|^{2} \\
& +\frac{\left\|\theta_{1}^{*}\right\|^{2} M_{1}}{a_{11}}+\frac{\bar{\varepsilon}_{1}^{2}}{2 a_{12}}+\frac{l_{1}^{2}}{2 a_{12}}
\end{aligned}
$$

Then, substituting $\alpha_{1}$ and $\dot{\theta}_{1}$ into 29 , one has

$\dot{V}_{1} \leq-\mu_{1} \tilde{X}^{\mathrm{T}} \tilde{X}-c_{1} \eta_{1}^{2}+\Xi \eta_{1} \eta_{2}+\frac{\sigma_{1}}{\pi_{1}} \tilde{\theta}_{1}^{\mathrm{T}} \dot{\theta}_{1}+\Lambda_{1}$

where $\Lambda_{1}=a_{14}\|P \delta\|^{2} / 2+\left\|\theta_{1}^{*}\right\|^{2} M_{1} / a_{11}+\bar{\varepsilon}_{1}^{2} /\left(2 a_{12}\right)$ $+i_{1}^{2} /\left(2 a_{12}\right)$ and $\mu_{1}=\lambda_{\min }(Q)-1 /\left(2 a_{13}\right)-1 /\left(2 a_{14}\right)>0$.

Step 2: The derivative of $\eta_{2}$ is

$$
\begin{aligned}
\dot{\eta}_{2}= & \dot{z}_{2}-\dot{\xi}_{2} \\
= & \hat{x}_{3}+\hat{h}_{2}\left(\hat{X} \mid \theta_{2}\right)+l_{2} \tilde{x}_{1}-\dot{\lambda}_{2}-\dot{\xi}_{2} \\
= & z_{3}+\lambda_{3}+\theta_{2}^{*^{\mathrm{T}}} \phi_{2}(\hat{X})-\tilde{\theta}_{2}^{\mathrm{T}} \phi_{2}(\hat{X})+l_{2} \tilde{x}_{1}-\dot{\lambda}_{2} \\
& -\dot{\xi}_{2}-\theta_{2}^{*^{\mathrm{T}}} \phi_{2}\left(\hat{X}_{2}\right)+\theta_{2}^{\mathrm{T}} \phi_{2}\left(\hat{X}_{2}\right)+\tilde{\theta}_{2}^{\mathrm{T}} \phi_{2}\left(\hat{X}_{2}\right)
\end{aligned}
$$

where $\tilde{\theta}_{2}=\theta_{2}^{*}-\theta_{2}$. The Lyapunov function is constructed as

$V_{2}=V_{1}+\frac{1}{2} \eta_{2}^{2}+\frac{1}{2 \pi_{2}} \tilde{\theta}_{2}^{2}$

Then we have

$$
\begin{aligned}
\dot{V}_{2}= & \dot{V}_{1}+\eta_{2} \dot{\eta}_{2}-\frac{1}{\pi_{2}} \tilde{\theta}_{2}^{\mathrm{T}} \dot{\theta}_{2} \\
= & \dot{V}_{1}-\frac{1}{\pi_{2}} \tilde{\theta}_{2}^{\mathrm{T}} \dot{\theta}_{2}+\eta_{2}\left(\eta_{3}+\alpha_{2}+\theta_{2}^{*^{\mathrm{T}}} \phi_{2}(\hat{X})\right. \\
& -\tilde{\theta}_{2}^{\mathrm{T}} \phi_{2}(\hat{X})-\theta_{2}^{*^{\mathrm{T}}} \phi_{2}\left(\hat{X}_{2}\right)+\theta_{2}^{\mathrm{T}} \phi_{2}\left(\hat{X}_{2}\right) \\
& +\tilde{\theta}_{2}^{\mathrm{T}} \phi_{2}\left(\hat{X}_{2}\right)+l_{2} \tilde{x}_{1}-\dot{\lambda}_{2}+c_{2} \xi_{2}+\Xi \xi_{1} \\
& \left.+l_{2} \operatorname{sign}\left(\xi_{2}\right)\right) .
\end{aligned}
$$

Similar to step 1, we can obtain

$\eta_{2}\left(\theta_{2}^{*^{\mathrm{T}}} \phi_{2}(\hat{X})-\theta_{2}^{*^{\mathrm{T}}} \phi_{2}\left(\hat{X}_{2}\right)\right) \leq a_{21} \eta_{2}^{2}+\frac{\left\|\theta_{2}^{*}\right\|^{2} M_{2}}{a_{21}}$

$\eta_{2} \iota_{2} \operatorname{sign}\left(\xi_{2}\right) \leq \frac{a_{22}}{2} \eta_{2}^{2}+\frac{\imath_{2}^{2}}{2 a_{22}}$

$-\eta_{2} \tilde{\theta}_{2}^{\mathrm{T}} \phi_{2}(\hat{X}) \leq \frac{a_{23}}{2} \eta_{2}^{2}+\frac{\tilde{\theta}_{2}^{\mathrm{T}} \tilde{\theta}_{2} M_{2}}{2 a_{23}}$ 
Substituting (34)-36) into (33), yields

$$
\begin{aligned}
\dot{V}_{2} \leq & -\mu_{1} \tilde{X}^{\mathrm{T}} \tilde{X}-c_{1} \eta_{1}^{2}+\frac{\sigma_{1}}{\pi_{1}} \tilde{\theta}_{1}^{\mathrm{T}} \dot{\theta}_{1}+\Lambda_{1}+\eta_{2} \eta_{3} \\
& +\eta_{2}\left(\alpha_{2}+a_{21} \eta_{2}+\frac{a_{23}}{2} \eta_{2}+\frac{a_{22}}{2} \eta_{2}\right. \\
& \left.+\theta_{2}^{\mathrm{T}} \phi_{2}\left(\hat{X}_{2}\right)+l_{2} \tilde{x}_{1}-\dot{\lambda}_{2}+c_{2} \xi_{2}+\Xi_{z_{1}}\right) \\
& +\eta_{2} \tilde{\theta}_{2}^{\mathrm{T}} \phi_{2}\left(\hat{X}_{2}\right)-\frac{1}{\pi_{2}} \tilde{\theta}_{2}^{\mathrm{T}} \dot{\theta}_{2}+\frac{\tilde{\theta}_{2}^{\mathrm{T}} \tilde{\theta}_{2} M_{2}}{2 a_{23}} \\
& +\frac{\left\|\theta_{2}^{*}\right\|^{2} M_{2}}{a_{21}}+\frac{l_{2}^{2}}{2 a_{22}}
\end{aligned}
$$

Then, substituting $\alpha_{2}$ and $\dot{\theta}_{2}$ into [37), one has

$$
\begin{aligned}
\dot{V}_{2} \leq & -\mu_{1} \tilde{X}^{\mathrm{T}} \tilde{X}-c_{1} \eta_{1}^{2}-c_{2} \eta_{2}^{2}+\eta_{2} \eta_{3} \\
& +\frac{\sigma_{1}}{\pi_{1}} \tilde{\theta}_{1}^{\mathrm{T}} \theta_{1}+\frac{\sigma_{2}}{\pi_{2}} \tilde{\theta}_{2}^{\mathrm{T}} \theta_{2}+\frac{\tilde{\theta}_{2}^{\mathrm{T}} \tilde{\theta}_{2} M_{2}}{2 a_{23}}+\Lambda_{2}
\end{aligned}
$$

where $\Lambda_{2}=\Lambda_{1}+\left\|\theta_{2}^{*}\right\|^{2} M_{2} / a_{21}+l_{2}^{2} /\left(2 a_{22}\right)$.

Step $k(k=3, \ldots, n-1)$ : The derivative of $\eta_{k}$ is

$$
\begin{aligned}
\dot{\eta}_{k}= & \dot{z}_{k}-\dot{\xi}_{k} \\
= & \hat{x}_{k+1}+\hat{h}_{k}\left(\hat{X} \mid \theta_{k}\right)+l_{k} \tilde{x}_{1}-\dot{\lambda}_{k}-\dot{\xi}_{k} \\
= & z_{k+1}+\lambda_{k+1}+\theta_{k}^{*^{\mathrm{T}}} \phi_{k}(\hat{X})-\tilde{\theta}_{k}^{\mathrm{T}} \phi_{k}(\hat{X})+l_{k} \tilde{x}_{1} \\
& -\dot{\lambda}_{k}-\dot{\xi}_{k}-\theta_{k}^{*^{\mathrm{T}}} \phi_{k}\left(\hat{X}_{k}\right)+\theta_{k}^{\mathrm{T}} \phi_{k}\left(\hat{X}_{k}\right) \\
& +\tilde{\theta}_{k}^{\mathrm{T}} \phi_{k}\left(\hat{X}_{k}\right)
\end{aligned}
$$

where $\tilde{\theta}_{k}=\theta_{k}^{*}-\theta_{k}$. The Lyapunov function is constructed as

$$
V_{k}=V_{k-1}+\frac{1}{2} \eta_{k}^{2}+\frac{1}{2 \pi_{k}} \tilde{\theta}_{k}^{2}
$$

Then we have

$$
\begin{aligned}
\dot{V}_{k}= & \dot{V}_{k-1}+\eta_{k} \dot{\eta}_{k}-\frac{1}{\pi_{k}} \tilde{\theta}_{k}^{\mathrm{T}} \dot{\theta}_{k} \\
= & \dot{V}_{k-1}-\frac{1}{\pi_{k}} \tilde{\theta}_{k}^{\mathrm{T}} \dot{\theta}_{k} \\
& +\eta_{k}\left(\eta_{k+1}+\alpha_{k}+\theta_{k}^{*^{\mathrm{T}}} \phi_{k}(\hat{X})-\tilde{\theta}_{k}^{\mathrm{T}} \phi_{k}(\hat{X})\right. \\
& -\theta_{k}^{*^{\mathrm{T}}} \phi_{k}\left(\hat{X}_{k}\right)+\theta_{k}^{\mathrm{T}} \phi_{k}\left(\hat{X}_{k}\right)+\tilde{\theta}_{k}^{\mathrm{T}} \phi_{k}\left(\hat{X}_{k}\right) \\
& \left.+l_{k} \tilde{x}_{1}-\dot{\lambda}_{k}+c_{k} \xi_{k}+\xi_{k-1}+\imath_{k} \operatorname{sign}\left(\xi_{k}\right)\right) .
\end{aligned}
$$

Similar to step 2, we can obtain

$$
\begin{aligned}
& \eta_{k}\left(\theta_{k}^{*^{\mathrm{T}}} \phi_{k}(\hat{X})-\theta_{k}^{*^{\mathrm{T}}} \phi_{k}\left(\hat{X}_{k}\right)\right) \leq a_{k 1} \eta_{k}^{2}+\frac{\left\|\theta_{k}^{*}\right\|^{2} M_{k}}{a_{k 1}} \\
& \eta_{k} \iota_{k} \operatorname{sign}\left(\xi_{k}\right) \leq \frac{a_{k 2}}{2} \eta_{k}^{2}+\frac{\imath_{k}^{2}}{2 a_{k 2}} \\
& -\eta_{k} \tilde{\theta}_{k}^{\mathrm{T}} \phi_{k}(\hat{X}) \leq \frac{a_{k 3}}{2} \eta_{k}^{2}+\frac{\tilde{\theta}_{k}^{\mathrm{T}} \tilde{\theta}_{k} M_{k}}{2 a_{k 3}}
\end{aligned}
$$

Substituting 42, -44) into 41, yields

$$
\begin{aligned}
\dot{V}_{k} \leq & \dot{V}_{k-1}+\eta_{k} \eta_{k+1}+\eta_{k}\left(\alpha_{k}+a_{k 1} \eta_{k}+\frac{a_{k 3}}{2} \eta_{k}\right. \\
& +\frac{a_{k 2}}{2} \eta_{k}+\theta_{k}^{\mathrm{T}} \phi_{k}\left(\hat{X}_{k}\right)+l_{k} \tilde{x}_{1}-\dot{\lambda}_{k}+c_{k} \xi_{k} \\
& \left.+\xi_{k-1}\right)+\eta_{k} \tilde{\theta}_{k}^{\mathrm{T}} \phi_{k}\left(\hat{X}_{k}\right)-\frac{1}{\pi_{k}} \tilde{\theta}_{k}^{\mathrm{T}} \dot{\theta}_{k} \\
& +\frac{\tilde{\theta}_{k}^{\mathrm{T}} \tilde{\theta}_{k} M_{k}}{2 a_{k 3}}+\frac{\left\|\theta_{k}^{*}\right\|^{2} M_{k}}{a_{k 1}}+\frac{l_{k}^{2}}{2 a_{k 2}}
\end{aligned}
$$

Then, substituting $\alpha_{k}$ and $\dot{\theta}_{k}$ into 45 , one has

$$
\begin{aligned}
\dot{V}_{k} \leq & -\mu_{1} \tilde{X}^{\mathrm{T}} \tilde{X}-\sum_{i=1}^{k} c_{i} \eta_{i}^{2}+\eta_{k} \eta_{k+1}+\sum_{i=1}^{k} \frac{\sigma_{i}}{\pi_{i}} \tilde{\theta}_{i}^{\mathrm{T}} \theta_{i} \\
& +\sum_{i=2}^{k} \frac{\tilde{\theta}_{i}^{\mathrm{T}} \tilde{\theta}_{i} M_{i}}{2 a_{i 3}}+\Lambda_{k}
\end{aligned}
$$

where $\Lambda_{k}=\Lambda_{k-1}+\left\|\theta_{k}^{*}\right\|^{2} M_{k} / a_{k 1}+i_{k}^{2} /\left(2 a_{k 2}\right)$.

Remark 10 It can be noticed that the function $\hat{h}_{k}\left(\hat{X} \mid \theta_{k}\right)$ in the neural observer (13) contains the whole state estimate $\hat{X}=\left[\hat{x}_{1}, \ldots, \hat{x}_{n}\right]^{\mathrm{T}}$. To ensure that only partial state estimate $\hat{X}_{k}=\left[\hat{x}_{1}, \ldots, \hat{x}_{k}\right]^{\mathrm{T}}$ is included in the virtual controller $\alpha_{k}$, we employ Young's inequality and the structural property of RBF to deal with the whole variable $\hat{X}$, which solves the problem of algebraic loop. Compared with [15]-[16] and [12], the method in this article neither needs the restrictive condition that the unknown function satisfies monotonically increasing property, nor needs to introduce additional lowpass filter.

Step $n$ : The actual controller will be given in this final step, the derivative of $\eta_{n}$ is

$$
\begin{aligned}
\dot{\eta}_{n}= & \dot{z}_{n}-\dot{\xi}_{n} \\
= & u+\hat{h}_{n}\left(\hat{X} \mid \theta_{n}\right)+l_{n} \tilde{x}_{1}-\dot{\lambda}_{n}-\dot{\xi}_{n} \\
= & u+\theta_{n}^{\mathrm{T}} \phi_{n}(\hat{X})-\tilde{\theta}_{n}^{\mathrm{T}} \phi_{n}(\hat{X})+\tilde{\theta}_{n}^{\mathrm{T}} \phi_{n}(\hat{X}) \\
& +l_{n} \tilde{x}_{1}-\dot{\lambda}_{n}-\dot{\xi}_{n} .
\end{aligned}
$$

Choose the Lyapunov function

$V_{n}=V_{n-1}+\frac{1}{2} \eta_{n}^{2}+\frac{1}{2 \pi_{n}} \tilde{\theta}_{n}^{2}$.

Then we have

$$
\begin{aligned}
\dot{V}_{n}= & \dot{V}_{n-1}+\eta_{n} \dot{\eta}_{n}-\frac{1}{\pi_{n}} \tilde{\theta}_{n}^{\mathrm{T}} \dot{\theta}_{n} \\
= & \dot{V}_{n-1}-\frac{1}{\pi_{n}} \tilde{\theta}_{n}^{\mathrm{T}} \dot{\theta}_{n}+\eta_{n} \tilde{\theta}_{n}^{\mathrm{T}} \phi_{n}(\hat{X}) \\
& +\eta_{n}\left(u+\theta_{n}^{\mathrm{T}} \phi_{n}(\hat{X})-\tilde{\theta}_{n}^{\mathrm{T}} \phi_{n}(\hat{X})+l_{n} \tilde{x}_{1}\right. \\
& \left.-\dot{\lambda}_{n}+c_{n} \xi_{n}+\xi_{n-1}+\imath_{n} \operatorname{sign}\left(\xi_{n}\right)\right) .
\end{aligned}
$$


Applying Young's inequality yields

$\eta_{n} l_{n} \operatorname{sign}\left(\xi_{n}\right) \leq \frac{a_{n 2}}{2} \eta_{n}^{2}+\frac{l_{n}^{2}}{2 a_{n 2}}$

$-\eta_{n} \tilde{\theta}_{n}^{\mathrm{T}} \phi_{n}(\hat{X}) \leq \frac{a_{n 3}}{2} \eta_{n}^{2}+\frac{\tilde{\theta}_{n}^{\mathrm{T}} \tilde{\theta}_{n} M_{n}}{2 a_{n 3}}$.

Substituting (50)-51] into 49], yields

$$
\begin{aligned}
\dot{V}_{n} \leq & \dot{V}_{n-1}+\eta_{n}\left(u+\frac{a_{n 3}}{2} \eta_{n}+\frac{a_{n 2}}{2} \eta_{n}+\theta_{n}^{\mathrm{T}} \phi_{n}(\hat{X})\right. \\
& \left.+l_{n} \tilde{x}_{1}-\dot{\lambda}_{n}+c_{n} \xi_{n}+\xi_{n-1}\right)+\eta_{n} \tilde{\theta}_{n}^{\mathrm{T}} \phi_{n}(\hat{X}) \\
& -\frac{1}{\pi_{n}} \tilde{\theta}_{n}^{\mathrm{T}} \dot{\theta}_{n}+\frac{\tilde{\theta}_{n}^{\mathrm{T}} \tilde{\theta}_{n} M_{n}}{2 a_{n 3}}+\frac{\imath_{n}^{2}}{2 a_{n 2}} .
\end{aligned}
$$

In the interval $t \in\left[t_{q}, t_{q+1}\right)$, from 10$]$,we know that

$|\tau(t)-u(t)|<\rho_{a x}$ ing

So, there is a time-varying parameter $|\kappa(t)| \leq 1$ satisfiy-

$u(t)=\tau(t)-\kappa(t) \rho_{a x}$

By substituting $\tau(t)$ and (54) into (52), we have

$$
\begin{aligned}
\dot{V}_{n} & \leq \dot{V}_{n-1}+\eta_{n}\left(\alpha_{n}-\bar{\rho} \tanh \left(\frac{\eta_{n} \bar{\rho}}{\varpi}\right)-\kappa(t) \rho_{a x}\right. \\
& +\frac{a_{n 3}}{2} \eta_{n}+\frac{a_{n 2}}{2} \eta_{n}+\theta_{n}^{\mathrm{T}} \phi_{n}(\hat{X})+l_{n} \tilde{x}_{1} \\
& \left.-\dot{\lambda}_{n}+c_{n} \xi_{n}+\xi_{n-1}\right)+\eta_{n} \tilde{\theta}_{n}^{\mathrm{T}} \phi_{n}(\hat{X}) \\
& -\frac{1}{\pi_{n}} \tilde{\theta}_{n}^{\mathrm{T}} \dot{\theta}_{n}+\frac{\tilde{\theta}_{n}^{\mathrm{T}} \tilde{\theta}_{n} M_{n}}{2 a_{n 3}}+\frac{\imath_{n}^{2}}{2 a_{n 2}} .
\end{aligned}
$$

Substitute $\alpha_{n}$ and $\dot{\theta}_{n}$ into $(55)$, yields

$$
\begin{aligned}
\dot{V}_{n} \leq & -\mu_{1} \tilde{X}^{\mathrm{T}} \tilde{X}-\sum_{k=1}^{n} c_{k} \eta_{k}^{2}+\sum_{k=1}^{n} \frac{\sigma_{k}}{\pi_{k}} \tilde{\theta}_{k}^{\mathrm{T}} \theta_{k} \\
& +\sum_{k=2}^{n} \frac{\tilde{\theta}_{k}^{\mathrm{T}} \tilde{\theta}_{k} M_{k}}{2 a_{k 3}}+\Lambda_{n}+\eta_{n}\left(-\bar{\rho} \tanh \left(\frac{\eta_{n} \bar{\rho}}{\bar{\omega}}\right)\right. \\
& \left.-\kappa(t) \rho_{a x}\right)
\end{aligned}
$$

where $\Lambda_{n}=\Lambda_{n-1}+i_{n}^{2} /\left(2 a_{n 2}\right)$. From $\tilde{\theta}_{k}=\theta_{k}^{*}-\theta_{k}$, one has

$\tilde{\theta}_{k}^{\mathrm{T}} \theta_{k} \leq \frac{1}{2} \theta_{k}^{*^{\mathrm{T}}} \theta_{k}^{*}-\frac{1}{2} \tilde{\theta}_{k}^{\mathrm{T}} \tilde{\theta}_{k}$

then

$\sum_{k=1}^{n} \frac{\sigma_{k}}{\pi_{k}} \tilde{\theta}_{k}^{\mathrm{T}} \theta_{k} \leq-\sum_{k=1}^{n} \frac{\sigma_{k}}{2 \pi_{k}} \tilde{\theta}_{k}^{\mathrm{T}} \tilde{\theta}_{k}+\sum_{k=1}^{n} \frac{\sigma_{k}}{2 \pi_{k}} \theta_{k}^{*^{\mathrm{T}}} \theta_{k}^{*}$
Based on Lemma 1, $\bar{\rho}>\rho_{a x}$ and $|\kappa(t)| \leq 1$, from 56 and (58), we have

$$
\begin{aligned}
\dot{V}_{n} \leq & -\mu_{1} \tilde{X}^{\mathrm{T}} \tilde{X}-\sum_{k=1}^{n} c_{k} \eta_{k}^{2}+\sum_{k=1}^{n} \frac{\sigma_{k}}{\pi_{k}} \tilde{\theta}_{k}^{\mathrm{T}} \theta_{k} \\
& +\sum_{k=2}^{n} \frac{\tilde{\theta}_{k}^{\mathrm{T}} \tilde{\theta}_{k} M_{k}}{2 a_{k 3}}+\Lambda_{n}-\eta_{n} \bar{\rho} \tanh \left(\frac{\eta_{n} \bar{\rho}}{\bar{\sigma}}\right) \\
& +\left|\eta_{n} \bar{\rho}\right| \\
\leq & -\mu_{1} \tilde{X}^{\mathrm{T}} \tilde{X}-\sum_{k=1}^{n} c_{k} \eta_{k}^{2}-\left(\frac{\sigma_{1}}{2 \pi_{1}} \tilde{\theta}_{1}^{\mathrm{T}} \tilde{\theta}_{1}+\right. \\
& \left.\sum_{k=2}^{n}\left(\frac{\sigma_{k}}{2 \pi_{k}}-\frac{M_{k}}{2 a_{k 3}}\right) \tilde{\theta}_{k}^{\mathrm{T}} \tilde{\theta}_{k}\right)+\Lambda_{n}^{\prime}
\end{aligned}
$$

where $\Lambda^{\prime}{ }_{n}=\Lambda_{n}+\sum_{k=1}^{n} \sigma_{k} \theta_{k}^{*^{\mathrm{T}}} \theta_{k}^{*} / 2 \pi_{k}+0.2785 \varpi$

According to the above analysis, the main result of this article is stated as follows.

Theorem 1 For the nonstrict-feedback uncertain nonlinear systems (1) with Assumption 1, design the neural state observer (13), the command filtered system (17), error compensation system (21), the virtual controllers and actual controller (18), the adaptive updating laws (20), then the proposed event-triggered control strategy can guarantee that the tracking error converges to a prescribed small region near zero within preset finite time $t_{r}$ at predesigned converging mode during the period $t \in\left[0, t_{r}\right)$ and all the signals $x_{k}, \hat{x}_{k}, z_{k}, \eta_{k}, \xi_{k}, \alpha_{k}, k=1, \ldots, n, \tau(t)$ and $u(t)$ are bounded. In addition, $\forall q \in z^{+}$, there is a positve instant $t^{\aleph}>0$ satisfying $\left\{t_{q+1}-t_{q}\right\} \geq t^{\aleph}$.

Proof :To prove the stability of the error compensation system 21, we choose the Lyapunov function as $V_{\xi}=$ $\sum_{k=1}^{n} \xi_{k}^{2} / 2$. Then, the derivative of $V_{\xi}$ is obtained as

$$
\begin{aligned}
\dot{V}_{\xi}= & -c_{1} \xi_{1}^{2}+\Xi \xi_{1} \xi_{2}+\Xi\left(\lambda_{2}-\alpha_{1}\right) \xi_{1}-\Xi \iota_{1}\left|\xi_{1}\right| \\
& -c_{2} \xi_{2}^{2}-\Xi \xi_{1} \xi_{2}+\left(\lambda_{3}-\alpha_{2}\right) \xi_{2}+\xi_{2} \xi_{3} \\
& -\iota_{2}\left|\xi_{2}\right|+\cdots-c_{k} \xi_{k}^{2}-\xi_{k-1} \xi_{k} \\
& +\left(\lambda_{k+1}-\alpha_{k}\right) \xi_{k}+\xi_{k} \xi_{k+1}-\imath_{k}\left|\xi_{k}\right| \\
& +\cdots-c_{n} \xi_{n}^{2}-\xi_{n-1} \xi_{n}-\imath_{n}\left|\xi_{n}\right| \\
= & -\sum_{k=1}^{n} c_{k} \xi_{k}^{2}-\Xi\left(\imath_{1}\left|\xi_{1}\right|-\left(\lambda_{2}-\alpha_{1}\right) \xi_{1}\right) \\
& -\sum_{k=2}^{n-1}\left(\imath_{k}\left|\xi_{k}\right|-\left(\lambda_{k+1}-\alpha_{k}\right) \xi_{k}\right)-\imath_{n}\left|\xi_{n}\right|
\end{aligned}
$$

Similar to [44] and [45], we can get that $\left|\left(\lambda_{k+1}-\alpha_{k}\right)\right| \leq$ $\beta_{k}, k=1, \ldots, n-1$, can be achieved with the positive constant $\beta_{k}$. Since $\Xi>0$, via choosing suitable parameter $l_{k}>$ 
$\beta_{k}$, one has

$\dot{V}_{\xi} \leq-\sum_{k=1}^{n} c_{k} \xi_{k}^{2}$

To analysis the stability of the closed-loop system, we construct the whole Lyapunov function as

$V=V_{n}+V_{\xi}$

The derivative of $V$ is obtained as

$$
\begin{aligned}
\dot{V} \leq & -\frac{2 \mu_{1}}{\lambda_{\max }(P)} \frac{\tilde{X}^{\mathrm{T}} P \tilde{X}}{2}-2 \sum_{k=1}^{n} c_{k} \frac{\eta_{k}^{2}}{2} \\
& -\left(\sigma_{1} \frac{\tilde{\theta}_{1}^{\mathrm{T}} \tilde{\theta}_{1}}{2 \pi_{1}}+\sum_{k=2}^{n}\left(\sigma_{k}-\frac{2 \pi_{k} M_{k}}{2 a_{k 3}}\right) \frac{\tilde{\theta}_{k}^{\mathrm{T}} \tilde{\theta}_{k}}{2 \pi_{k}}\right) \\
& -2 \sum_{k=1}^{n} c_{k} \frac{\xi_{k}^{2}}{2}+\Lambda^{\prime}{ }_{n} \\
\leq & -\Theta V+\Lambda^{\prime}{ }_{n}
\end{aligned}
$$

where $\Theta=\min \left\{2 \mu_{1} / \lambda_{\max }(P), 2 c_{k}, \sigma_{1}, \sigma_{k}-2 \pi_{k} M_{k} /\left(2 a_{k 3}\right)\right\}$. Furthermore, select the design parameters $\sigma_{k}, \pi_{k}, a_{k 3}$ so that $\sigma_{k}-2 \pi_{k} M_{k} /\left(2 a_{k 3}\right)>0$. Consequently, from (63), we have

$V(t) \leq V(0) e^{-\Theta t}+\frac{\Lambda_{n}^{\prime}}{\Theta}$

Then it can be obtained that $\eta_{k}, \tilde{\theta}_{k}, \xi_{k}$ and $\theta_{k}, k=$ $1, \ldots, n$ are bounded. From $z_{k}=\eta_{k}+\xi_{k}, z_{k}$ is bounded. Next, $x_{1}$ and $\hat{x}_{1}$ are bounded as $y_{d}$ is bounded, which implies that $\alpha_{1}$ and $\lambda_{2}$ are bounded. Similarly, we can prove $x_{k}, \hat{x}_{k}, \alpha_{k}, \tau(t)$ and $u(t)$ are also bounded. Therefore, all the signals inside the closed-loop system are bounded.

Furthermore, since $z_{1}=s_{1}$ is bounded, we obtain

$$
\begin{aligned}
\left|\chi_{1}\right| & <\left|\frac{1}{\mu}\right| \\
& = \begin{cases}\left|\frac{\left(1-\mu_{t_{r}}\right)\left(t_{r}-t\right)^{3} \mu_{0}+\mu_{0} \mu_{t_{r}} t_{r}^{3}}{t_{r}^{3}}\right|, t \in\left[0, t_{r}\right) \\
\mu_{0} \mu_{t_{r}}, & t \in\left[t_{r},+\infty\right)\end{cases}
\end{aligned}
$$

which implies that the error $\left|\chi_{1}\right|$ reduces to $\mu_{0} \mu_{t_{r}}$ within a pregiven time $t_{r}$ at predesigned converging mode governed by $\left(1-\mu_{t_{r}}\right)\left(t_{r}-t\right)^{3} \mu_{0} / t_{r}^{3}$.

Next, we prove that there exists $t^{\aleph}>0$ such that $\left\{t_{q+1}-\right.$ $\left.t_{q}\right\} \geq t^{\aleph}, \forall q \in z^{+}$. Based on $\varsigma(t)=\tau(t)-u(t), \forall t \in\left[t_{q}, t_{q+1}\right)$, one has

$$
\begin{aligned}
\frac{\mathrm{d}}{\mathrm{d} t}|\varsigma(t)| & =\frac{\mathrm{d}}{\mathrm{d} t} \sqrt{\varsigma(t) \cdot \zeta(t)} \\
& =\operatorname{sign}(\varsigma(t)) \dot{\zeta}(t) \\
& \leq|\dot{\tau}(t)| .
\end{aligned}
$$

From (18), we can demonstrate that $\tau(t)$ is differentiable and its time derivative $\dot{\tau}(t)$ is a function with all bounded signals, which implies that there is a positive constant $\gamma>0$ satisfiying $|\dot{\tau}(t)| \leq \gamma$. In addition, $\varsigma\left(t_{q}\right)=0$ and $\lim _{t \rightarrow q+1} \varsigma(t) \geq$ $\rho_{\text {in }}$, so the lower bound of event-triggered time intervals $t^{\aleph}$ must satisfy $t^{\aleph} \geq \rho_{\text {in }} / \gamma>0$. Therefore, the Zeno-behaviour is ruled out.

Remark 11 It can be demonstrated from (65) that in the interval $t \in\left[t_{q}, t_{q+1}\right)$, if the value of $\mu_{0}$ determined by the initial tracking error $\chi_{1}(0)$ does not change, then the converging rate of $\left|\chi_{1}\right|$ is affected not only by $\mu_{t_{r}}$ but also by $t_{r}$. A faster tracking process can be achieved by decreasing $\mu_{t_{r}}$ or shortening preset time $t_{r}$, which will be shown in the later simulation comparison experiment.

Remark 12 The developed event-triggered output feedback control strategy can obtain given performance specifications for the tracking error. Such solution, very useful in practical engineering, has never been proposed for the uncertain nonstrict-feeback systems, as described in (1).

\section{Simulation Examples}

This section is to demonstrate the effectiveness and superiority of the developed control strategy by two examples.

Example 1: Consider the nonstrict-feedback nonlinear system as follows

$\left\{\begin{array}{l}\dot{x}_{1}=x_{2}+h_{1}(X) \\ \dot{x}_{2}=u+h_{2}(X) \\ y=x_{1}\end{array}\right.$

where $h_{1}(X)=x_{2} e^{-0.5 x_{1}}, h_{2}(X)=x_{1} \sin \left(x_{2}^{2}\right)$. The initial states are given as $x_{1}(0)=2, x_{2}(0)=0, \hat{x}_{1}(0)=1.5$ and $\hat{x}_{1}(0)=-2$. The desired reference signal is $y_{d}=\sin (t)$.

Construct the following neural state observer

$\left\{\begin{array}{c}\dot{\hat{x}}_{1}=\hat{x}_{2}+l_{1}\left(y-\hat{x}_{1}\right)+\theta_{1}^{\mathrm{T}} \phi_{1}(\hat{X}) \\ \dot{\hat{x}}_{2}=u+l_{2}\left(y-\hat{x}_{1}\right)+\theta_{2}^{\mathrm{T}} \phi_{2}(\hat{X})\end{array}\right.$

where the observer gain vector is designed as $L=\left[l_{1}, l_{2}\right]=$ $[29.9643,113.4592] . \theta_{1}^{\mathrm{T}} \phi_{1}(\hat{X})$ and $\theta_{2}^{\mathrm{T}} \phi_{2}(\hat{X})$ contain 125 nodes and 75 nodes respectively. For each of two neural networks, centers are spaced evenly in $[-1,2] \times[-2,1.5]$ and widths are equal to 3.85 . The parameter update laws are given as

$\dot{\theta}_{1}=\pi_{1} \Xi \eta_{1} \phi_{1}\left(\hat{x}_{1}\right)-\sigma_{1} \theta_{1}$

$\dot{\theta}_{2}=\pi_{2} \eta_{2} \phi_{2}(\hat{X})-\sigma_{2} \theta_{2}$

with $\theta_{1}(0)=0_{125 \times 1}$ and $\theta_{2}(0)=0_{75 \times 1}$. 
Then, we design virtual controllers, actual controller and event-triggered mechanism as

$$
\begin{aligned}
\alpha_{1}= & -\frac{c_{1} z_{1}}{\Xi}-\frac{a_{13}}{2} \eta_{1} \Xi-a_{11} \eta_{1} \Xi-a_{12} \eta_{1} \Xi \\
& -\theta_{1}^{\mathrm{T}} \phi_{1}\left(\hat{x}_{1}\right)-\frac{2 \dot{\mu}}{\pi \mu^{2}} \arctan s_{1}+\dot{y}_{d} \\
\alpha_{2}= & -c_{2} z_{2}-\frac{a_{23}}{2} \eta_{2}-\frac{a_{22}}{2} \eta_{2}-\theta_{2}^{\mathrm{T}} \phi_{2}(\hat{X}) \\
& -l_{2} \tilde{x}_{1}+\dot{\lambda}_{2}-\Xi z_{1} \\
\tau(t)= & \alpha_{2}-\bar{\rho} \tanh \left(\frac{\eta_{2} \bar{\rho}}{\varpi}\right) \\
u(t)= & \tau\left(t_{q}\right) \\
t_{q+1}= & \left\{\begin{array}{l}
\inf \left\{t>t_{q}|| \varsigma(t) \mid \geq \rho_{a x}\right\}, \text { if } t<T^{\dagger} \\
\inf \left\{t>t_{q}|| \varsigma(t) \mid \geq \rho_{i n}\right\}, \text { if } t \geq T^{\dagger}
\end{array}\right.
\end{aligned}
$$

where the command filter system is given as

$\omega_{1} \dot{\lambda}_{2}+\lambda_{2}=\alpha_{1}, \lambda_{2}(0)=\alpha_{1}(0)$

and the error compensation mechanism is constructed as

$\dot{\xi}_{1}=-c_{1} \xi_{1}+\Xi \xi_{2}+\Xi\left(\lambda_{2}-\alpha_{1}\right)-\Xi \imath_{1} \operatorname{sign}\left(\xi_{1}\right)$

$\dot{\xi}_{2}=-c_{2} \xi_{2}-\Xi \xi_{1}-\imath_{2} \operatorname{sign}\left(\xi_{2}\right)$

with $\xi_{k}(0)=0(k=1,2)$.

In the simulation, choosing the design parameters as $c_{1}=0.1$, $c_{2}=5, \bar{\rho}=9, \varpi=15, \omega_{1}=0.01, \imath_{1}=l_{2}=0.1, t_{r}=5, \mu_{t r}=0.05, \mu_{0}=2.5$, $T^{\dagger}=1, \rho_{a x}=8, \rho_{i n}=0.2, \pi_{1}=\pi_{2}=0.01, \sigma_{1}=\sigma_{2}=1, a_{11}=a_{12}=0.025$, $a_{13}=0.1, a_{23}=0.9, a_{22}=0.1$.

To show the effectiveness of event-triggered mechanism, comparative simulations between the fixed threshold approach, relative threshold strategy and the presented method are carried out. The fixed threshold approach can be obtain from [33] and it is given as follows:

$$
\begin{aligned}
& \tau(t)=\alpha_{2}-\bar{\rho} \tanh \left(\frac{\eta_{2} \bar{\rho}}{\varpi}\right) \\
& u(t)=\tau\left(t_{q}\right) \\
& t_{q+1}=\inf \left\{t>t_{q}|| \varsigma(t) \mid \geq \rho_{\text {fix }}\right\}
\end{aligned}
$$

where $\bar{\rho}=9$ and the value of $\rho_{\text {fix }}$ is selected as $0.2,4.2$ and 8 , respectively, for comparative simulations.

The relative threshold strategy from [34] is given as follows:

$$
\begin{aligned}
& \tau_{r e l}(t)=-\left(1+\sigma_{r e l}\right)\left(\alpha_{2} \tanh \left(\frac{\eta_{2} \alpha_{2}}{\bar{\varpi}_{r e l}}\right)\right. \\
&\left.+\bar{\rho}_{r e l} \tanh \left(\frac{\eta_{2} \bar{\rho}_{r e l}}{\bar{\varpi}_{r e l}}\right)\right) \\
& u_{r e l}(t)= \tau_{r e l}\left(t_{q}\right) \\
& t_{q+1}=\inf \left\{t>t_{q}|| \varsigma(t)\left|\geq \sigma_{r e l}\right| u_{r e l}(t) \mid+\rho_{r e l}\right\}
\end{aligned}
$$

where $\bar{\rho}_{\text {rel }}=0.09, \sigma_{r e l}=0.4, \varpi_{r e l}=5$ and $\rho_{\text {rel }}=0.05$.

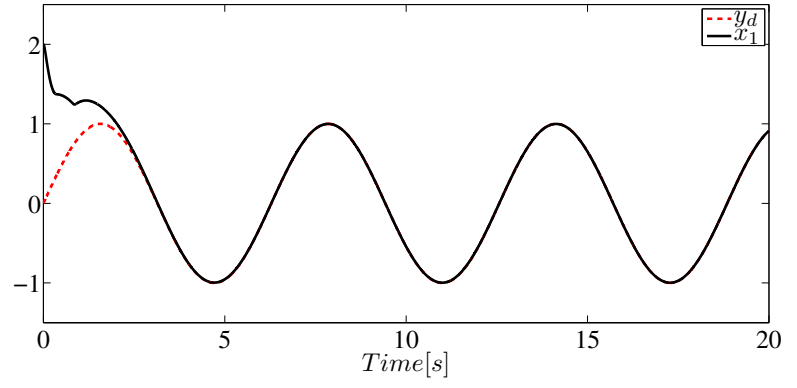

Fig. 2 Trajectories of $y_{d}$ and $x_{1}$ in Example 1.

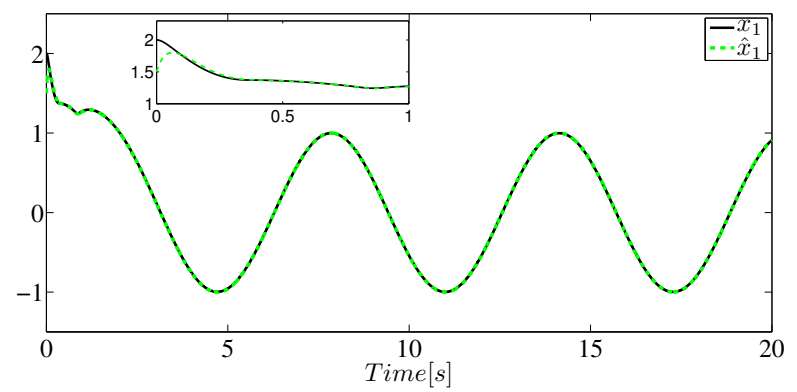

Fig. 3 Trajectories of $x_{1}$ and $\hat{x}_{1}$ in Example 1.

Figs. 2, 8 describe simulation results, where Fig. 5 shows that the tracking error $\chi_{1}$ converges to a small region around zero within a preset time $t_{r}=5$ at predesigned decay rate. It also points out that the proposed method can obtain superior tracking performance according to faster response and smaller oscillation. For the proposed method, Fig. 3 and Fig. 4 show the system state and estimation of them, the control signal is shown in Fig. 6 and the trajectories of the norm of the adaptive parameters $\left\|\theta_{1}\right\|$ and $\left\|\theta_{2}\right\|$ are shown in Fig. 7 and Fig. 8 ,

Furthermore, performance comparisons of the presented method, the fixed threshold approach and relative threshold strategy are summarized in Table I. The integrated time absolute and integrated absolute error in [46], labelled as ITAE and IAE, are employed to evaluate dynamic performance and stable precision of tracking controllers, respectively, where ITAE $=\int_{0}^{20} v\left|\chi_{1}(v)\right| \mathrm{d} v$ and IAE $=\int_{0}^{20}\left|\chi_{1}(v)\right| \mathrm{d} v$.

Table I demonstrates that the presented method is superior to the other two event-triggered mechanisms since it has the lower ITAE and IAE while requiring less triggering numbers.

Example 2: To verify the effectiveness of the proposed controller in the practical system, the network-based onelink manipulator obtained from [47] is considered as follows:

$J \ddot{v}+F_{v} \dot{v}+G \sin (v)=\tau_{v}$

where $\dot{v}$ and $v$ are the velocity and position of the rigid link, respectively, $F_{v}=1 \mathrm{~N} \cdot \mathrm{s} / \mathrm{rad}$ denotes the overall damping 


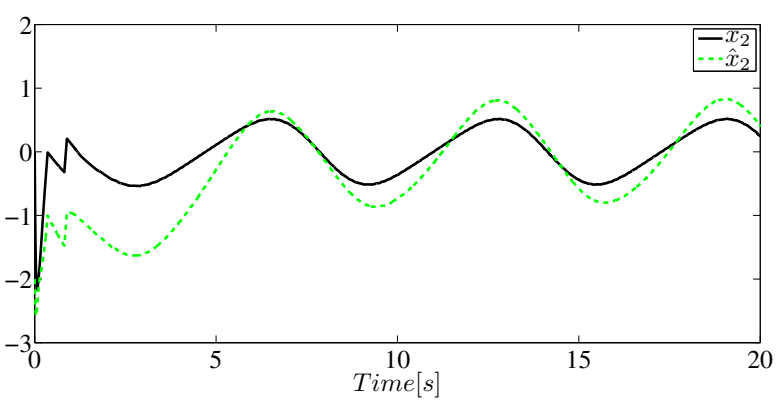

Fig. 4 Trajectories of $x_{2}$ and $\hat{x}_{2}$ in Example 1.

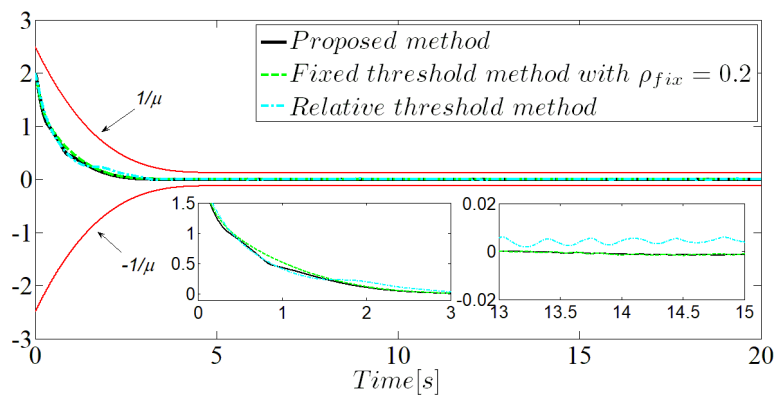

Fig. 5 Trajectories of $\chi_{1}$ under different methods.

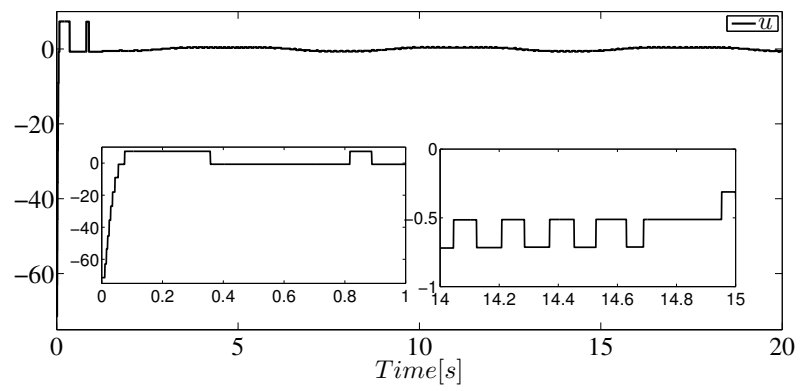

Fig. 6 Trajectory of controller $u$ in Example 1.

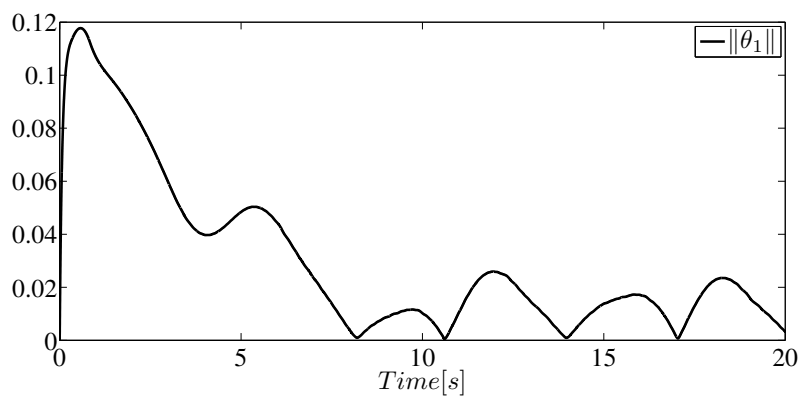

Fig. 7 Trajectory of $\left\|\theta_{1}\right\|$ in Example 1.

coefficient, $G$ denotes the gravity, $J=1 \mathrm{~kg} \cdot \mathrm{m}^{2}$ denotes the rotational inertia of motor.

Define $u=\tau_{v}, x_{1}=v$, and $x_{2}=\dot{v}$, from manipulator system (75), one has

$$
\left\{\begin{array}{l}
\dot{x}_{1}=x_{2}+h_{1}(X) \\
\dot{x}_{2}=u+h_{2}(X)
\end{array}\right.
$$

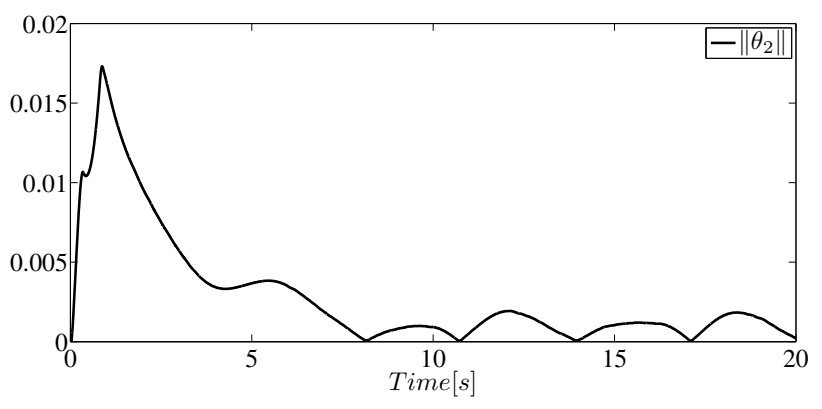

Fig. 8 Trajectory of $\left\|\theta_{2}\right\|$ in Example 1.

Table 1 Performance comparisons of different methods

\begin{tabular}{cccc}
\hline Method & ITAE & IAE & Triggering numbers \\
\hline Proposed method & 1.08 & 1.29 & 227 \\
Fixed threshold with $\rho_{\text {fix }}=0.2$ & 1.18 & 1.39 & 354 \\
Fixed threshold with $\rho_{\text {fix }}=4.2$ & 1.46 & 1.43 & 232 \\
Fixed threshold with $\rho_{\text {fix }}=8$ & 1.56 & 1.31 & 235 \\
Relative threshold & 1.79 & 1.43 & 236 \\
\hline
\end{tabular}

where $h_{1}(X)=0.2 \sin \left(x_{1}\right) \cos \left(x_{2}\right)$ and $h_{2}(X)=-x_{2}-10 \sin \left(x_{1}\right)$. In (76), we intentionally introduce disturbed term $h_{1}(X)$. The desired reference signal is $y_{d}=0.3 \sin (t)$.

In the simulation, choose the design parameters as $l_{1}=$ $20, l_{2}=200, c_{1}=0.1, c_{2}=5, \bar{\rho}=9, \varpi=15, \omega_{1}=0.01, l_{1}=l_{2}=0.1$, $t_{r}=2, \mu_{t r}=0.02, \mu_{0}=2.4, T^{\dagger}=1, \rho_{a x}=8, \rho_{i n}=0.2, \pi_{1}=0.01, \pi_{2}=0.02$, $\sigma_{1}=\sigma_{2}=1, a_{11}=a_{12}=0.025, a_{13}=0.1, a_{23}=0.9, a_{22}=0.1 . \theta_{1}^{\mathrm{T}} \phi_{1}(\hat{X})$ and $\theta_{2}^{\mathrm{T}} \phi_{2}(\hat{X})$ contain 20 nodes and 30 nodes respectively. For each of two neural networks, centers are spaced evenly in $[-2.5,0.5] \times[-1,7]$ and widths are equal to 8.8 . The initial values are set as $x_{1}(0)=-2, x_{2}(0)=0, \hat{x}_{1}(0)=-1.5$, $\hat{x}_{2}(0)=1, \xi_{k}(0)=0(k=1,2), \theta_{1}(0)=0_{20 \times 1}$ and $\theta_{2}(0)=$ $0_{30 \times 1}$.

The simulation results are given in Figs. 9,15 , where the tracking performance and tracking error are shown in Fig. 9 and Fig. 12 The system state and estimation of them are shown in Fig. 10 and Fig. 11 Fig. 13 describes the control signal. Fig. 14 and Fig. 15 show that the trajectories of the norm of the adaptive parameters $\left\|\theta_{1}\right\|$ and $\left\|\theta_{2}\right\|$ remain bounded.

Furthermore, we select different design parameters $t_{r}$ and $\mu_{t r}$ to demonstrate that these factors can affect the tracking performance via the proposed controller. The comparative results are given in Fig. 12, which is explained as follows.

1) For the design parameter $\mu_{t r}=0.1$, a faster error convergence rate can be achieved by selecting the smaller $t_{r}$, and for $t_{r}=2$, we can also obtain a faster transient process by choosing the smaller $\mu_{t r}$, which verifies the conclusion in Remark 11 .

2) Decreased the value of $\mu_{t r}$ will result in the better steady-state performance when $t_{r}=2$. 


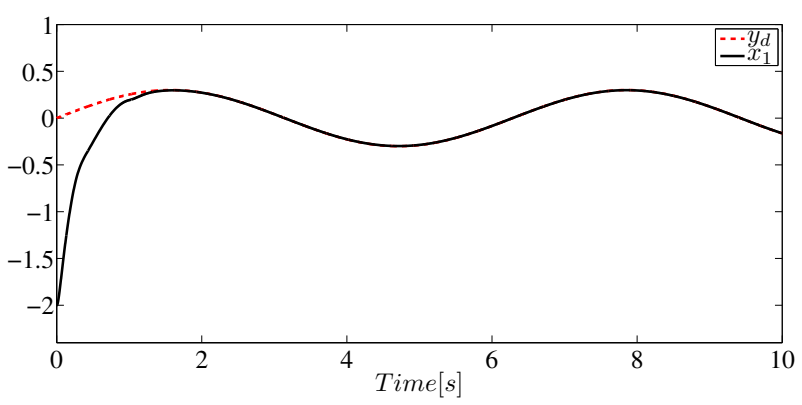

Fig. 9 Trajectories of $y_{d}$ and $x_{1}$ in Example 2.

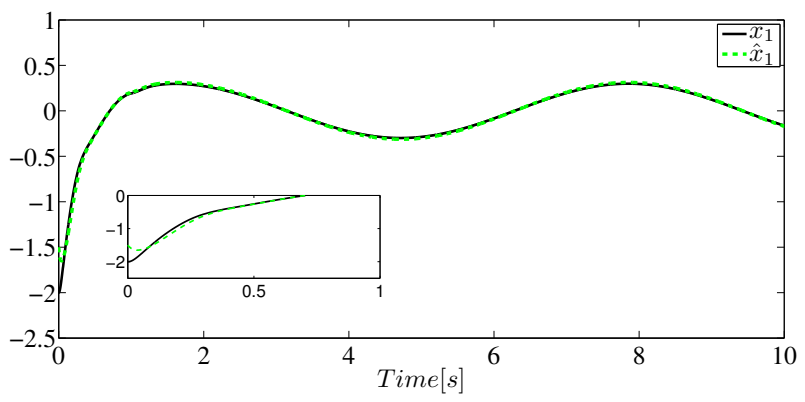

Fig. 10 Trajectories of $x_{1}$ and $\hat{x}_{1}$ in Example 2.

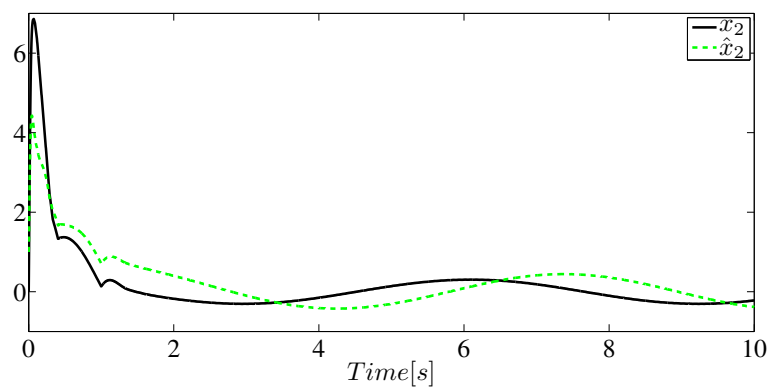

Fig. 11 Trajectories of $x_{2}$ and $\hat{x}_{2}$ in Example 2.

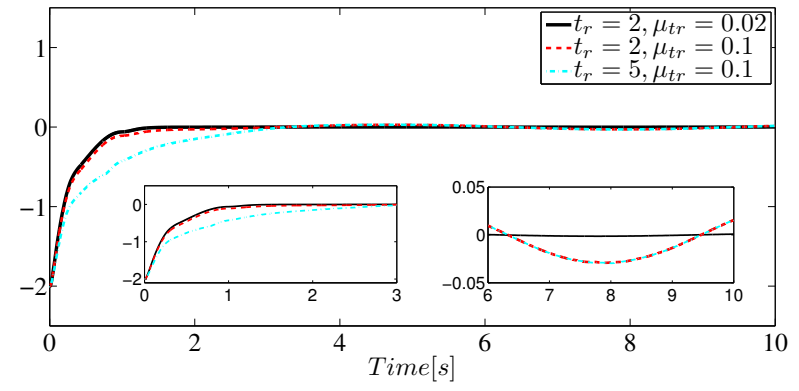

Fig. 12 Trajectories of $\chi_{1}$ under different cases.

\section{Conclusion}

This article has developed an event-triggered adaptive outputfeedback controller for nonstrict-feedback nonlinear systems with given performance specifications, where a novel eventtriggered mechanism with two thresholds and an improved speed transformation function have been designed. Neural state observer and neural networks have been adopted to ap-

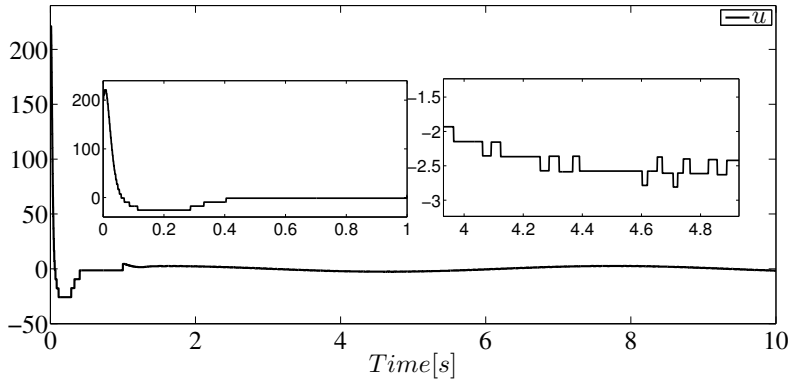

Fig. 13 Trajectory of controller $u$ in Example 2.

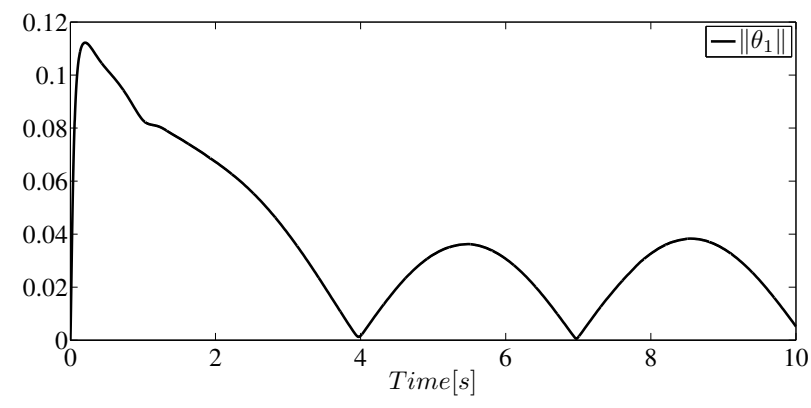

Fig. 14 Trajectory of $\left\|\theta_{1}\right\|$ in Example 2.

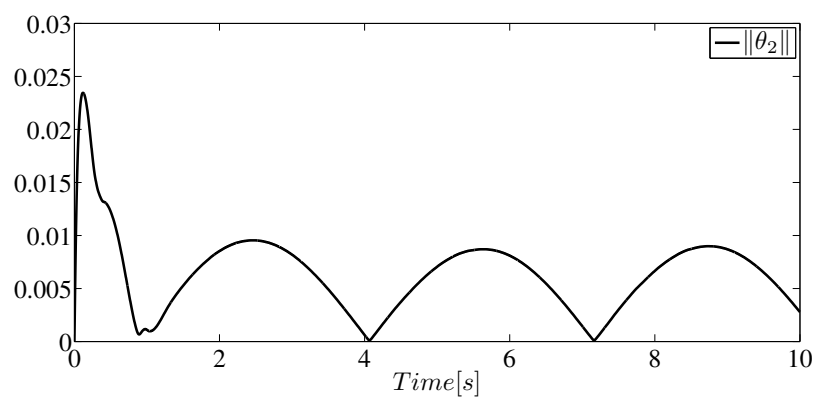

Fig. 15 Trajectory of $\left\|\theta_{2}\right\|$ in Example 2.

proximate unmeasured states and estimate uncertain functions, respectively. The structural property of RBF has been employed to deal with the algebraic loop problem caused by the nonstrict-feedback structure. Via flexibly incorporating command filtered technology, error compensation system with backstepping recursive method, a novel control strategy has been developed. All closed-loop signals are guaranteed to be bounded according to Lyapunov theory, and there is no Zeno-behavior. The developed control strategy can not only reduce the communication resources between the controller and actuator but also ensure that the output tracking error converges to a prescribed small region within preset time at predesigned converging rate. Finally, two simulation examples demonstrate the effectiveness and superiority of the developed control strategy. Further, we will extend the control strategy of this article to nonstrict-feedback switched nonlinear systems and nonstrict-feedback stochastic nonlinear systems. 


\section{Acknowledgment}

This work is support by the National Natural Science Foundation of China $(51879027,51579024)$

\section{Compliance with ethical standards}

Conflict of interest The authors declare that they have no conflict of interest.

Data availability statement The authors can confirm that all relevant data are included in the article.

\section{References}

1. Zerari, N., Chemachema, M.: Event-triggered adaptive outputfeedback neural-networks control for saturated strict-feedback nonlinear systems in the presence of external disturbance. Nonlinear Dyn. 4, 1343-1362 (2021)

2. Wang, L.B., Wang, H.Q., Liu, P.X.: Adaptive fuzzy finite-time control of stochastic nonlinear systems with actuator faults. Nonlinear Dyn. 104, 523-536 (2021)

3. Zhou, X., Gao, C., Li, Z.G., Ouyang, X.Y., Wu, L.B.: Observerbased adaptive fuzzy finite-time prescribed performance tracking control for strict-feedback systems with input dead-zone and saturation. Nonlinear Dyn. 103, 1645-1661 (2021)

4. Zhao, L., Yu, J.P., Wang, Q. G.: Finite-time tracking control for nonlinear systems via adaptive neural output feedback and command filtered backstepping. IEEE Trans. Neural Netw. Learn. Syst. 32(4), 1474-1485 (2021)

5. Sun, K.K., Liu, L., Qiu, J.B., Feng, G.: Fuzzy adaptive finite-time fault-tolerant control for strict-feedback nonlinear systems. IEEE Trans. Fuzzy Syst. 29(4), 786-796 (2021)

6. Tong, S.C., Min, X., Li, Y. X.: Observer-based adaptive fuzzy tracking control for strict-feedback nonlinear systems with unknown control gain functions. IEEE Trans. Cybern. 5(9), 3903-3913 (2020)

7. Yang, D., Hu, X., Liu, W. J., Guo, C.: Finite-time control design for course tracking of disturbed ships subject to input saturation. Int. J. Control (2020). https://doi.org/10.1080/00207179.2020.1856930

8. Ertugrul, T., Adli, M.A., Salamci, M.U.: Model reference adaptive control design for helicopters using gain scheduled reference models. In: Proceedings of 17th International Carpathian Control Conference. IEEE, pp. 194-203 (2016)

9. Joo, M.G., Lee, J.S.: A class of hierarchical fuzzy systems with constraints on the fuzzy rules. IEEE Trans. Fuzzy Syst. 13(2), 194-203 (2005)

10. Wang, A.Q., Liu, L., Qiu, J.B., Feng G.: Event-triggered adaptive fuzzy output-feedback control for nonstrict-feedback nonlinear systems with asymmetric output constraint. IEEE Trans. Cybern. (2020). https://doi.org/10.1109/TCYB.2020.2974775

11. Zou, A.M., Hou, Z.G., Tan, M.: Adaptive control of a class of nonlinear pure-feedback systems using fuzzy backstepping Approach. IEEE Trans. Fuzzy Syst. 16(4), 886-897 (2008)

12. Zhang, L. L., Yang, G.H.: Fault-estimation-based output-feedback adaptive FTC for uncertain nonlinear systems with actuator faults. IEEE Trans. Ind. Electron. 67(4), 3065-3075 (2020)

13. Chen, B., Liu, K., Liu, X., Shi, P., Lin, C., Zhang, H.: Approximation-based adaptive neural control design for a class of nonlinear systems. IEEE Trans. Cybern. 44(5), 610-619 (2014)
14. Chen, B., Lin, C., Liu, X.P., Liu, K.F.: Adaptive fuzzy tracking control for a class of MIMO nonlinear systems in nonstrictfeedback form. IEEE Trans. Cybern. 45(12), 2744-2755 (2015)

15. Wang, H., Liu, K., Liu, X., Chen, B., Lin, C.: Neuralbased adaptive output-feedback control for a class of nonstrictfeedback stochastic nonlinear systems. IEEE Trans. Cybern. 45(9), 1977-1987 (2015)

16. Chen, B., Zhang, H.G., Lin, C.: Observer-based adaptive neural network control for nonlinear systems in nonstrict-feedback form. IEEE Trans. Neural Netw. Learn. Syst. 27(1), 89-98 (2016)

17. Tong, S.C., Li, Y.M., Sui, S.: Adaptive fuzzy tracking control design for SISO uncertain nonstrict feedback nonlinear systems. IEEE Trans. Fuzzy Sys. 24(6), 1441-1454 (2016)

18. Chen, B., Zhang, H.G., Liu, X.P., Lin, C.: Neural Observer and Adaptive Neural Control Design for a Class of Nonlinear Systems. IEEE Trans. Neural Netw. Learn. Syst. 29(9), 4261-4271 (2018)

19. Li, Y.M., Shao, X.F., Tong, S.C.: Adaptive fuzzy prescribed performance control of nontriangular structure nonlinear systems. IEEE Trans. Fuzzy Sys. 28(10), 2416-2426 (2020)

20. Zhang, H.G., Liu, Y., Wang, Y.C.: Observer-based finite-time adaptive fuzzy control for nontriangular nonlinear systems with full-state constraints. IEEE Trans. Cybern. 51(3), 1110-1120 (2021)

21. Zhou, Q., Li, H., Wang, L., Lu, R.: Prescribed performance observer-based adaptive fuzzy control for nonstrict-feedback stochastic nonlinear systems. IEEE Trans. Syst., Man, Cybern., Syst. 48(10), 1747-1758 (2018)

22. Zhang J., Yang, G.H.: Prescribed performance fault-tolerant control of uncertain nonlinear systems with unknown control directions. IEEE Trans. Autom. Control 62(12), 6529-6239 (2017)

23. Li, Y., Tong, S., Liu, L., Feng, G.: Adaptive output-feedback control design with prescribed performance for switched nonlinear systems. Automatica 80, 225-231 (2017)

24. Sui, S., Chen, C.L.P., Tong, S.: Neural network filtering control design for nontriangular structure switched nonlinear systems in finite time. IEEE Trans. Neural Netw. Learn. Syst. 30(7), 2153-2162 (2019)

25. Sui, S., Tong, S., Chen, C.L.P.: Finite-time filter decentralized control for nonstrict-feedback nonlinear large-scale systems. IEEE Trans. Fuzzy Syst. 26(6), 3289-3300 (2018)

26. Zhao, Z., Jiang, Z.: Finite-time output feedback stabilization of lower-triangular nonlinear systems. Automatica 96, 259-269 (2018)

27. Zhao, K., Song, Y.D., Ma, T.D., He, L.: Prescribed performance control of uncertain Euler-Lagrange systems subject to fullstate constraints. IEEE Trans. Neural Netw. Learn. Syst. 29(8), 3478-3489 (2018)

28. Song, Y.D., Zhou, S.Y.: Neuroadaptive control with given performance specifications for MIMO strict-feedback systems under nonsmooth actuation and output constraints. IEEE Trans. Neural Netw. Learn. Syst. 29(9), 4414-4425 (2018)

29. Liu, C., G, C., Liu, X., Wang, H., Zhou, Y.: Adaptive finite-time prescribed performance control for stochastic nonlinear systems with unknown virtual control coefficients. Nonlinear Dyn. 104, 3655-3670 (2021)

30. Qiu, J.B., Wang, T., Sun, K.K., Rudas, I.J., Gao, H. J.: Disturbance observer-based adaptive fuzzy control for strict-feedback nonlinear systems with finite-time prescribed performance. IEEE Trans. Fuzzy Syst. (2021). https://doi.org/10.1109/TFUZZ.2021.3053327

31. Sun, W., Wu, Y.Q., Sun, Z.Y.: Command filter-based finite-time adaptive fuzzy control for uncertain nonlinear systems with prescribed performance. IEEE Trans. Fuzzy Syst. 28(12), 3161-3170 (2020)

32. Liu, Y., Liu, X.P., Jing, Y.W., Zhang, Z. Y.: A novel finite-time adaptive fuzzy tracking control scheme for nonstrict feedback systems. IEEE Trans. Fuzzy Syst. 27(4), 646-658 (2019) 
33. Qiu, J.B., Sun, K.K., Wang, T., Gao, H.J.: Observer-based fuzzy adaptive event-triggered control for pure-feedback nonlinear systems with prescribed performance. IEEE Trans. Fuzzy Syst. 27(11), 2152-2162 (2019)

34. Zhang C.H., Yang, G.H.: Event-triggered adaptive output feedback control for a class of uncertain nonlinear systems with actuator failures. IEEE Trans. Cybern. 50(1), 201-210 (2020)

35. Ma, J.L., Xu, S.Y., Ma, Q., Zhang, Z.Q.: Event-triggered adaptive neural network control for nonstrict-feedback nonlinear timedelay systems with unknown control directions. IEEE Trans. Neural Netw. Learn. Syst. 31(10), 4196-4025 (2020)

36. Nai, Y.Q., Yang, Q.Y., Wu, Z.Z.: Prescribed performance adaptive neural compensation control for intermittent actuator faults by state and output feedback. IEEE Trans. Neural Netw. Learn. Syst. (2020). https://doi.org/10.1109/TNNLS.2020.3026208

37. Xing, L., Wen, C., Liu, Z., Sun, H., Cai, J.: Adaptive compensation for actuator failures with event-triggered input. Automatica 85, 129-136 (2017)

38. Sanner, R.M., Slotine, J.-J.-E.: Gaussian networks for direct adaptive control. IEEE Trans. Neural Netw. 3(6), 837-863 (1992)

39. Zhang, L. L., Yang, G.H.: Adaptive fuzzy prescribed performance control of nonlinear systems with hysteretic actuator nonlinearity and faults. IEEE Trans. Syst., Man, Cybern., Syst. 48(10), $1747-1758(2018)$

40. Tong, S.C., Li, Y.M.: Observer-based fuzzy adaptive control for strict-feedback nonlinear systems. Fuzzy Sets Syst. 160(12), 1749-1764 (2009)

41. Liu, Y.J., Gong, M.Z., Tong, S.C., Chen, C.L.P., Li, D.J.: Adaptive fuzzy output feedback control for a class of nonlinear systems with full state constraints. IEEE Trans. Fuzzy Sys. 26(5), 2607-2617 (2018)

42. Liu, Y.J., Gong, M.Z., Liu, L., Tong, S.C., Chen, C.L.P.: Fuzzy observer constraint based on adaptive control for uncertain nonlinear MIMO systems with time-varying state constraints. IEEE Trans. Cybern. 51(3), 1308-1389 (2021)

43. Yu, J., Zhao, L., Yu, H., Lin, C.: Barrier Lyapunov functionsbased command filtered output feedback control for full-state constrained nonlinear systems. Automatica 105, 71-79 (2019)

44. Li, Y.X.: Finite time command filtered adaptive fault tolerant control for a class of uncertain nonlinear systems. Automatica 106, 117-123 (2019)

45. Sun, W., Su, S.F., Xia, J.W., Zhuang, G.M.: Command filter-based adaptive prescribed performance tracking control for stochastic uncertain nonlinear systems. IEEE Trans. Syst., Man, Cybern., Syst. (2020). https://doi.org/10.1109/TSMC.2019.2963220

46. Zheng, Z.W., Feroskhan, M.: Path following of a surface vessel with prescribed performance in the presence of input saturation and external disturbances. IEEE/ASME Trans. Mechatronics 22(6), 2564-2575 (2017)

47. Xing, L., Wen, C., Liu, Z., Su, H., Cai, J.: Event-triggered adaptive control for a class of uncertain nonlinear systems. IEEE Trans. Autom. Control 62(4), 2071-2076 (2017) 